\title{
Some new cases of realization of spectral multiplicity function for ergodic transformations
}

by

A. Katok (University Park, PA) and M. Lemańczyk (Toruń)

Abstract. Given a countable Abelian group $\mathbb{G}$, its automorphism $w$ for which $w^{M}=\mathrm{Id}$, and a subgroup $\mathbb{F} \subset \mathbb{G}$ we define

$$
M(\mathbb{G}, w, \mathbb{F})=\left\{\sharp\left(\left\{w^{i} \chi: i \in \mathbb{Z}\right\} \cap \mathbb{F}\right): \chi \in \mathbb{F} \backslash\{0\}\right\} .
$$

We prove that each finite set of the form $M(\mathbb{G}, w, \mathbb{F}) \cup\{2\}$ is realized as the set of essential values of the multiplicity function of the Koopman operator of some weakly mixing automorphism.

Introduction. Assume that $T$ is an ergodic automorphism of a standard probability Borel space $(X, \mathcal{B}, \mu)$. The corresponding unitary operator $U_{T}$ : $L^{2}(X, \mathcal{B}, \mu) \rightarrow L^{2}(X, \mathcal{B}, \mu), U_{T}(f)=f \circ T$, is called the Koopman operator and is usually considered only on $L_{0}^{2}(X, \mathcal{B}, \mu)$, that is, on the subspace of zero mean functions.

Two basic questions of the spectral theory of Koopman representations are:

1. What measures appear as the maximal spectral type of an ergodic automorphism?

2. What subsets of $\mathbb{N}^{*} \cup\{\infty\}$ appear as the set of essential values of the multiplicity function of an ergodic transformation? We will call such subsets Koopman realizable or simply realizable.

Those questions do not exhaust the spectral theory and in Section 6 we will mention some other problems.

Only obvious restrictions are known for the first question, namely density, symmetry and invariance under multiplication by eigenvalues, while many

2010 Mathematics Subject Classification: 37A05, 28D05, 37A30, 47A10.

Key words and phrases: ergodicity, weak mixing, cocycle, spectrum, multiplicity function. 
more probably exist $\left({ }^{1}\right)$. However, for the second one it is expected that all subsets are realizable. It has already been shown that all subsets containing 1 are realizable [19] (reproved by a different argument in [3]). For more information on the subject see also earlier articles by Robinson [29], [30], and the surveys $[11,18]$. Less is known about Koopman realization of sets which do not contain 1. In [1] and [32] (see also the recent book by Anosov [6]) the Rokhlin problem of existence of a Koopman representation with a unique essential value (equal to $n, n \geq 2$ ) is solved for $n=2$. Moreover, Ageev [4] solved the Rokhlin problem in full generality. Danilenko [8] simplified the original construction of Ageev, and used it to show that sets of the form $n \cdot(\{1\} \cup F)$, where $F$ is an arbitrary subset of $\mathbb{N}^{*} \cup\{\infty\}$ without 1 , are Koopman realizable. Moreover, in [5] a new series of realizable sets, including the set $\{2,3, \ldots, n\}$, is given and those sets are also realizable in the class of mixing automorphisms.

In the present paper we make another modest step forward toward showing that many sets of integers are realizable (for the proof of the theorem below see Section 4).

Theorem 1 (Main Theorem). Let $\mathbb{G}$ be an arbitrary countable Abelian group $\mathbb{G}, w$ its automorphism for which $w^{M}=\mathrm{Id}$, and $\mathbb{F}$ a subgroup $\mathbb{G}$. Let

$$
\begin{aligned}
M(\mathbb{G}, w, \mathbb{F}) & :=\left\{\sharp\left(\left\{w^{i} \chi: i \in \mathbb{Z}\right\} \cap \mathbb{F}\right): \chi \in \mathbb{F} \backslash\{0\}\right\} \\
& =\left\{\sharp\left(\left\{w^{i} \eta: i \in \mathbb{Z}\right\} \cap \mathbb{F}\right): \eta \in \mathbb{G} \backslash\{0\}\right\} \backslash\{0\} .
\end{aligned}
$$

Then there exists a weakly mixing automorphism $T$ of a Lebesgue space $(X, \mu)$ for which the set of essential values of spectral multiplicities in $L_{0}^{2}(X, \mathcal{B}, \mu)$ is equal to $M(\mathbb{G}, w, \mathbb{F}) \cup\{2\}$.

Our method builds upon the solution of the Rokhlin problem for $n=2$ in $[1,32]$ and the methods of periodic approximation theory summarized in [16]. We widely use the presence of various operators in the weak closure of the iterates of a Koopman operator or its restriction to a particular subspace to assert that no "unexpected" overlaps appear between various components of the spectrum and thus guarantee that the spectral multiplicity can be deduced from a certain algebraic picture.

In order to deduce from our construction that all finite subsets containing 2 are Koopman realizable one needs to prove an algebraic lemma that each finite subset of $\mathbb{N}$ is of the form $M(\mathbb{G}, w, \mathbb{F})$. The construction in the proof of the Algebraic Lemma in [19] gives rise to sets $M(\mathbb{G}, w, \mathbb{F})$ which contain 1.

$\left({ }^{1}\right)$ This is an instance of a wide-spread phenomenon in ergodic theory: there are precious few "real theorems" and plenty of examples and counterexamples, many of which may be disguised as theorems; the principal result of the present paper is not an exception. 
We show, however, that all sets $\{2, n\}$ are Koopman realizable, which answers a question from [33].

We believe that the techniques developed in this paper may be useful in the further investigation of the spectral multiplicity problem. This is one of the reasons we do not aim at particular brevity in calculations and try to present all ingredients in sufficient detail.

1. Spectral multiplicities of tensor products. Let $V$ be a unitary operator on a separable Hilbert space $H$. If $f \in H$, then we denote by $\mathbb{Z}_{V}(f)$ (or simply $\mathbb{Z}(f)$ if no confusion arises) the cyclic space generated by $f$, that is, the closure of the span of $\left\{V^{n} f: n \in \mathbb{Z}\right\}$. The spectral measure $\sigma_{f}=\sigma_{V, f}$ of $f \in H$ is the (positive, finite) measure on $\mathbb{T}=\{z \in \mathbb{C}:|z|=1\}$ given by

$$
\widehat{\sigma}_{f}(n)=\left\langle V^{n} f, f\right\rangle \quad \text { for all } n \in \mathbb{Z} \text {. }
$$

The operator $V$ is determined up to unitary isomorphism by its maximal spectral type $\sigma_{V}$ (the equivalence class of a spectral measure which dominates all spectral measures) and a Borel function $M_{V}: \mathbb{T} \rightarrow \mathbb{N}^{*} \cup\{\infty\}$ defined $\sigma_{V}$-a.e. and called the multiplicity function (see e.g. the Appendix in [28] or [18] for more information on the spectral theory of unitary operators). Each member of the essential range of $M_{V}$ is called an essential value of the multiplicity function.

If $V: H \rightarrow H$ is a unitary operator on a Hilbert space, $F \subset H$ is a closed $V$-invariant subspace and $V^{n_{t}} \rightarrow A$ weakly ( $A$ is a bounded linear operator on $H$ ) then $A F \subset F$. This follows immediately from the fact that a closed subspace is weakly closed.

Lemma 1. Let $V_{i}: H_{i} \rightarrow H_{i}, i=1,2$, be unitary operators with simple spectrum. Assume moreover that

(i) $V_{i}^{n_{t}} \rightarrow \frac{1}{2}\left(\mathrm{Id}+V_{i}\right)$ weakly, $i=1,2$,

(ii) $V_{i}^{m_{t}} \rightarrow \frac{1}{2}\left(\mathrm{Id}+c_{i} V_{i}\right)$ weakly, $i=1,2$.

If $c_{1} \neq c_{2}$ then $V_{1} \otimes V_{2}$ also has a simple spectrum.

Proof. Assume that $H_{i}=\mathbb{Z}_{V_{i}}\left(f_{i}\right), i=1,2$, and let $F:=\mathbb{Z}_{V_{1} \otimes V_{2}}\left(f_{1} \otimes f_{2}\right)$. We will show that

$$
H_{1} \otimes H_{2}=F
$$

We have

$$
V_{1}^{k} f_{1} \otimes V_{2}^{k} f_{2} \in F
$$

for each $k \in \mathbb{Z}$. Since

$$
\left(V_{1} \otimes V_{2}\right)^{n_{t}} \rightarrow \frac{1}{4}\left(\mathrm{Id}+V_{1}\right) \otimes\left(\mathrm{Id}+V_{2}\right), \quad\left(f_{1}+V_{1} f_{1}\right) \otimes\left(f_{2}+V_{2} f_{2}\right) \in F,
$$

using (2) we obtain

$$
f_{1} \otimes V_{2} f_{2}+V_{1} f_{1} \otimes f_{2} \in F .
$$


Similarly, $\left(f_{1}+c_{1} V_{1} f_{1}\right) \otimes\left(f_{2}+c_{2} V_{2} f_{2}\right) \in F$, whence

$$
c_{1} V_{1} f_{1} \otimes f_{2}+f_{1} \otimes\left(c_{2} V_{2} f_{2}\right) \in F .
$$

It follows from (3) and (4) that $f_{1} \otimes V_{2} f_{2} \in F$ and $V_{1} f_{1} \otimes f_{2} \in F$. Therefore $F$ is invariant under $V_{1} \otimes \mathrm{Id}$ and $\mathrm{Id} \otimes V_{2}$, and (1) follows.

Lemma 2 ([3], [6], [16], [32]). Let $V: H \rightarrow H$ be a unitary operator with simple continuous spectrum. If the weak closure of powers of $V$ contains $\frac{1}{2}(\mathrm{Id}+V)$, then $V \otimes V$ has uniform spectral multiplicity 2 .

Proof. Recall that if $V_{1}, V_{2}$ are unitary operators then the maximal spectral type of their tensor product is the convolution of the spectral types of the factors, i.e. $\sigma_{V_{1} \otimes V_{2}}=\sigma_{V_{1}} * \sigma_{V_{2}}$. Moreover, if the spectra of $V_{i}$ are simple, then $M_{V_{1} \otimes V_{2}}(c)$ is (for $\sigma_{V_{1}} * \sigma_{V_{2}}$-a.a. $c \in \mathbb{T}$ ) either equal to the number of atoms of the measure $\eta_{c}$, where

$$
\sigma_{V_{1}} \otimes \sigma_{V_{2}}=\int_{\mathbb{T}} \eta_{c} \otimes \delta_{c} d\left(\sigma_{V_{1}} * \sigma_{V_{2}}\right)(c),
$$

if $\eta_{c}$ is purely atomic, or equal to infinity.

In view of the continuity of the spectrum, $\sigma \otimes \sigma(\{(z, z): z \in \mathbb{T}\})=0$, and thus the multiplicities of $V \otimes V$ are even. Then as in the proof of Lemma 1 one checks that $f \otimes V f$ and $V f \otimes f V \otimes V$-generate the space $H \otimes H$ for each $V$-cyclic vector $f$ for $H$.

Let $H$ be a Hilbert space. Given $k \geq 1$, let $\mathcal{F}_{k} \subset H^{\otimes k}$ be the subspace of symmetric $k$-tensors; it is usually denoted by $H^{\odot k}$ and consists of the tensors invariant under the group $\Sigma_{k}$ of all coordinate permutations. Denote by $\operatorname{Sym}\left(f_{1} \otimes \cdots \otimes f_{k}\right)$ the vector $\sum_{\tau \in \Sigma_{k}} f_{\tau(1)} \otimes \cdots \otimes f_{\tau(k)}$. Notice that $\operatorname{Sym}\left(f_{1} \otimes \cdots \otimes f_{k}\right)$ is equal (up to a multiplicative constant) to the value of the orthogonal projection $\pi_{H \odot k}$ on $H^{\odot k}$ at $f_{1} \otimes \cdots \otimes f_{k}$. If $V$ is a unitary operator on $H$ then we denote by $e^{V}$ the corresponding unitary operator on the symmetric Fock space $\bigoplus_{k \geq 0} H^{\odot k}$, that is, $e^{V}=\bigoplus_{k \geq 0} V^{\odot k}$, and $V^{\odot k}$ is the restriction of $V^{\otimes k}$ to $\mathcal{F}_{k}, k \geq 0$. Recall that the symmetric tensor product $H^{\odot n}$ can also be defined abstractly by putting

$$
\left\langle x_{1} \odot \cdots \odot x_{n}, y_{1} \odot \cdots \odot y_{n}\right\rangle=\sum_{\tau \in \Sigma_{n}}\left\langle x_{1}, y_{\tau(1)}\right\rangle \cdot \cdots \cdot\left\langle x_{n}, y_{\tau(n)}\right\rangle
$$

and then $\operatorname{Sym}\left(f_{1} \otimes \cdots \otimes f_{k}\right)=\sqrt{k !} f_{1} \odot \cdots \odot f_{k}$.

Below we will consider unitary operators $V$ with simple continuous spectrum such that the weak closure of powers of $V$ contains $\frac{1}{2}(\kappa \cdot \operatorname{Id}+V)$ for infinitely many $\kappa$ with $|\kappa| \leq 1$. Notice that such operators have continuous spectrum. Indeed, if $V f=c f$, where necessarily $|c|=1$, then $c^{n_{t}} \rightarrow \frac{1}{2}(\kappa+c)$, which is possible only if $\kappa=c$. Ageev [2, proof of Theorem 1] shows that the operator $e^{V}$ has a simple spectrum. In fact, Ageev considers operators for which $\sigma_{V}=\sigma_{V}^{\prime}+\delta_{1}$ where $\sigma_{V}^{\prime}$ is continuous. This is natural when we are 
studying multiplicities of the Koopman representations associated to Cartesian products $T^{\times n}$ since in the computation of the spectral multiplicities for $L_{0}^{2}\left(X^{\times n}, \mu^{\otimes n}\right)$ we must take into account the subspace of constant functions for $U_{T}$. Therefore, formally speaking, the proposition below is not a generalization of Ageev's result since on the nontrivial subspace of fixed points of $V$ the weak convergences may hold only if $\kappa=1$. Our proof is based on a different idea than in [2] and gives rise to a slightly more general result. Proposition 1 will be applied to invariant subspaces of some Koopman representations and in such a case, in general, the $\kappa$ s may not even be real numbers.

Proposition 1. Assume that $V$ and $W$ are unitary operators with simple spectrum defined on separable Hilbert spaces $H$ and $G$ respectively. Assume that $S \subset \mathbb{C}$ is countable. Assume moreover that for each $\kappa \in S$ there exist subsequences $\left(n_{t}^{(\kappa)}\right),\left(m_{t}^{(\kappa)}\right)$ and $\widetilde{\kappa}$ satisfying $\widetilde{\kappa}^{q} \neq \kappa^{q}$ for each $q \geq 1$ such that we have the following weak convergences:

$$
\begin{array}{rlrl}
V^{n_{t}^{(\kappa)}} & \rightarrow \frac{1}{2}(\kappa \cdot \mathrm{Id}+V), \quad W^{n_{t}^{(\kappa)}} & \rightarrow \frac{1}{2}(\kappa \cdot \mathrm{Id}+W), \\
V_{t}^{m_{t}^{(\kappa)}} & \rightarrow \frac{1}{2}(\kappa \cdot \mathrm{Id}+V), \quad W^{m_{t}^{(\kappa)}} \rightarrow \frac{1}{2}(\widetilde{\kappa} \cdot \mathrm{Id}+W) .
\end{array}
$$

Then $e^{V} \otimes W$ has a simple spectrum.

Proof. Fix $k \geq 1$. We need to show that $\left(\left.V^{\otimes k}\right|_{\mathcal{F}_{k}}\right) \otimes W$ has a simple spectrum. Assume that $H=\mathbb{Z}_{V}(f)$ and $G=\mathbb{Z}_{W}(g)$ for some $f \in H$ and $g \in G$. We intend to show that

$$
H^{\odot k} \otimes G=\mathbb{Z}_{V \odot k \otimes W}\left(f^{\otimes k} \otimes g\right) .
$$

We will first show that

$$
H^{\odot k}=\mathbb{Z}_{V \odot k}\left(f^{\otimes k}\right) .
$$

Define $F=\mathbb{Z}_{V \odot k}\left(f^{\otimes k}\right)$. The subspace $H^{\odot k}$ is $V^{\otimes k}$-invariant and $V^{\otimes k}$ is unitary; moreover, by (5) (or (6)) for each $\kappa \in S$,

$$
\left(V^{\otimes k}\right)^{n_{t}^{(\kappa)}} \rightarrow \frac{1}{2^{k}}(\kappa \cdot \mathrm{Id}+V)^{\otimes k},
$$

hence $H^{\odot k}$ is also invariant for the adjoint of $(\kappa \cdot \operatorname{Id}+V)^{\otimes k}$ and therefore

$$
\pi_{H \odot k} \circ(\kappa \cdot \operatorname{Id}+V)^{\otimes k}=(\kappa \cdot \operatorname{Id}+V)^{\otimes k} \circ \pi_{H \odot k} .
$$

We will now show by induction that

$$
\pi_{H \odot k}\left(f^{\otimes i_{0}} \otimes\left(V^{n_{1}} f\right)^{\otimes i_{1}} \otimes \cdots \otimes\left(V^{n_{p}} f\right)^{\otimes i_{p}}\right) \in F
$$

for all $i_{0}, \ldots, i_{p} \geq 0$ with $i_{0}+i_{1}+\cdots+i_{p}=k$, and $0=n_{0}<n_{1}<\cdots<n_{p}$.

Indeed, (10) is true for $p=0$ (that is, $n_{p}=0$ ). Assume its validity for all choices of admissible parameters when $n_{p} \leq N$. We will now prove that it also holds for the bound $N+1$. Assume that $j_{0}, \ldots, j_{p} \geq 0, \sum_{s=0}^{p} j_{s}=k$ and 
$\pi_{H \odot k}\left(f^{\otimes j_{0}} \otimes\left(V^{n_{1}} f\right)^{\otimes j_{1}} \otimes \cdots \otimes\left(V^{n_{p}} f\right)^{\otimes j_{p}}\right) \in F$. Since $F$ is $V^{\otimes k}$-invariant, it is also invariant for $(\kappa \cdot \operatorname{Id}+V)^{\otimes k}$ and its adjoint. Thus, in view of (9),

$$
\pi_{H \odot k}\left((\kappa \cdot \mathrm{Id}+V)^{\otimes k}\left(f^{\otimes j_{0}} \otimes\left(V^{n_{1}} f\right)^{\otimes j_{1}} \otimes \cdots \otimes\left(V^{n_{p}} f\right)^{\otimes j_{p}}\right) \in F .\right.
$$

In other words,

$$
\begin{aligned}
\pi_{H \odot k}\left(\kappa^{k} f^{\otimes j_{0}}\right. & \left.\otimes \cdots \otimes\left(V^{n_{p}} f\right)^{\otimes j_{p}}+\kappa^{k-1}(\ldots)+\cdots\right) \\
& =\kappa^{k} \pi_{H \odot k}\left(f^{\otimes j_{0}} \otimes \cdots \otimes\left(V^{n_{p}} f\right)^{\otimes j_{p}}\right)+\kappa^{k-1} \pi_{H \odot k}(\ldots)+\cdots \in F .
\end{aligned}
$$

We look at this relation as a certain polynomial equation, namely

$$
\kappa^{k}\left(\pi_{H \odot k}\left(f^{\otimes j_{0}} \otimes \cdots \otimes\left(V^{n_{p}} f\right)^{\otimes j_{p}}\right)+F\right)+\kappa^{k-1}\left(\pi_{H \odot k}(\ldots)+F\right)+\cdots=0
$$

in $H^{\odot k} / F$. Since the number of $\kappa$ s under consideration is infinite we infer that all "coefficients" from $H^{\odot k} / F$ vanish (indeed, it is enough to act on the above equalities by members of $\left.\left(H^{\odot k} / F\right)^{*}\right)$, that is, they all belong to $F$. We now focus on the coefficient of $\kappa^{k-1}$ for all admissible parameters. We obtain

$$
\begin{aligned}
& \pi_{H \odot k}(\underbrace{f \otimes \cdots \otimes f}_{\text {one } f \text { replaced by } V f} \otimes\left(V^{n_{1}} f\right)^{\otimes j_{1}} \otimes \cdots \otimes\left(V^{n_{p}} f\right)^{\otimes j_{p}} \\
& +f^{\otimes j_{0}} \otimes \underbrace{V^{n_{1}} f \otimes \cdots \otimes V^{n_{1}} f}_{\text {one } V^{n_{1}} f \text { replaced by } V^{n_{1}+1} f} \otimes \cdots \otimes\left(V^{n_{p}} f\right)^{\otimes j_{p}}+\cdots \\
& +f^{\otimes j_{0}} \otimes\left(V^{n_{1}} f\right)^{\otimes j_{1}} \otimes \cdots \otimes \underbrace{V^{n_{p}} f \otimes \cdots \otimes V^{n_{p}} f}_{\text {one } V^{n_{p}} f \text { replaced by } V^{n_{p}+1} f}) \in F .
\end{aligned}
$$

The linearity of $\pi_{H \odot k}$ and the induction assumption show that all summands except the last one are already in $F$. Hence

$$
\pi_{H \odot k}\left(f^{\otimes j_{0}} \otimes\left(V^{n_{1}} f\right)^{\otimes j_{1}} \otimes \cdots \otimes\left(V^{n_{p}} f\right)^{\otimes\left(j_{p}-1\right)} \otimes V^{n_{p}+1} f\right) \in F
$$

and we obtain (10) for the bound $N+1$ and all admissible parameters with the last element $j_{q}=1$. Let us now look at the coefficient of $\kappa^{k-2}$. We have

$$
\begin{aligned}
\pi_{H \odot k}\left(\sum_{u \leq w} f^{\otimes j_{1}} \otimes \cdots \otimes\right. & \underbrace{V^{n_{u}} f \otimes \cdots \otimes V^{n_{u}} f}_{\text {replace one } V^{n_{u}} f \text { by } V^{n_{u}+1} f} \otimes \cdots \\
& \otimes \underbrace{V^{n_{w}} f \otimes \cdots}_{\text {replace one } V^{n_{w}} f \text { by } V^{n_{w}+1} f} \otimes \cdots \otimes\left(V^{n_{p}} f\right)^{\otimes j_{p}}) \in F .
\end{aligned}
$$

If $w<p$ we may use the induction assumption: the corresponding symmetrizations are in $F$. This is still the case if $u<w=p$ : the corresponding symmetrizations are in $F$ because we have already proved (10) for $N+1$ and $j_{q}=1$. It follows that the only new case is when $j_{p} \geq 2(u=w=p)$; then

$$
\pi_{H \odot k}\left(f^{\otimes j_{0}} \otimes\left(V^{n_{1}} f\right)^{\otimes j_{1}} \otimes \cdots \otimes\left(V^{n_{p}} f\right)^{\otimes\left(j_{p}-2\right)} \otimes\left(V^{n_{p}+1} f\right)^{\otimes 2}\right) \in F
$$


and we obtain (10) for the bound $N+1$ with $j_{q}=2$. By considering the coefficients of $\kappa^{k-3}$ etc. we conclude that (10) holds.

Since $\mathbb{Z}_{V}(f)=H$,

$\operatorname{span}\left(\left\{f^{\otimes i_{0}} \otimes\left(V^{n_{1}} f\right)^{\otimes i_{1}} \otimes \cdots \otimes\left(V^{n_{p}} f\right)^{\otimes i_{p}}: i_{0}, \ldots, i_{p} \geq 0\right.\right.$,

$$
\left.\left.i_{0}+i_{1}+\cdots+i_{p}=k, n_{0}<n_{1}<\cdots<n_{p}, n_{i} \in \mathbb{Z}\right\}\right)=H^{\otimes k} .
$$

Thus, by (10), $F=\mathbb{Z}_{V \odot k}\left(f^{\otimes k}\right)=H^{\odot k}$ and hence $V^{\odot k}$ has a simple spectrum, that is, (8) follows.

Define $F_{1}=\mathbb{Z}_{V \odot k \otimes W}\left(f^{\otimes k} \otimes g\right)$. As before we find that for each $\kappa \in S$,

$$
(\kappa f+V f)^{\otimes k} \otimes(\kappa g+W g) \in F_{1}, \quad(\kappa f+V f)^{\otimes k} \otimes(\widetilde{\kappa} g+W g) \in F_{1},
$$

so by subtracting, $(\kappa f+V f)^{\otimes k} \otimes(\kappa-\widetilde{\kappa}) g \in F_{1}$ and hence $(\kappa f+V f)^{\otimes k} \otimes g$ $\in F_{1}$. By repeating our earlier argument for the coefficient of $\kappa^{0}$ we obtain

$$
(V f)^{\otimes k} \otimes g \in F_{1} .
$$

But $F_{1}$ is $(\kappa \cdot \operatorname{Id}+V)^{\otimes k} \otimes(\kappa \cdot \operatorname{Id}+W)-$ and $(\kappa \cdot \operatorname{Id}+V)^{\otimes k} \otimes(\widetilde{\kappa} \cdot \operatorname{Id}+W)$-invariant, so

$$
\left(\kappa^{2} V f+V^{2} f\right)^{\otimes k} \otimes\left(\kappa^{2} g+W g\right) \in F_{1}, \quad\left(\kappa^{2} V f+V^{2} f\right)^{\otimes k} \otimes\left(\widetilde{\kappa}^{2} g+W g\right) \in F_{1},
$$

whence $\left(\kappa^{2} V f+V^{2} f\right)^{\otimes k} \otimes g \in F_{1}$ and by repeating the same argument which led to (12), also $\left(V^{2} f\right)^{\otimes k} \otimes g \in F_{1}$, and by an easy induction $\left(V^{r} f\right)^{\otimes k} \otimes g \in F_{1}$ for each $r \geq 0$. Notice that the same holds for negative integers $r$, for if $V_{i}: H_{i} \rightarrow H_{i}$ is unitary $(i=1,2)$ and $V_{i}^{n_{t}} \rightarrow A_{i}$ weakly then directly from the definition of weak convergence it follows that $\left(V_{i}^{*}\right)^{n_{t}} \rightarrow A_{i}^{*}$ weakly, and also $\left(V_{1} \otimes V_{2}\right)^{n_{t}} \rightarrow A_{1} \otimes A_{2}$ weakly. The proof of (7) is now complete.

In order to prove that $e^{V}$ also has a cyclic vector we only need to show that for each $N \geq 1$,

$$
\mathbb{Z}_{\bigoplus_{k=1}^{N} V \odot k}\left(\sum_{k=1}^{N} f^{\otimes k}\right)=\bigoplus_{k=1}^{N} H^{\odot k} .
$$

Set

$$
\widetilde{F}=\mathbb{Z}_{\bigoplus_{k=1}^{N} V \odot k}\left(f^{\otimes k}\right) .
$$

As $\sum_{k=1}^{N} f^{\otimes k} \in \widetilde{F}$, also $\sum_{k=1}^{N}(\kappa f+V f)^{\otimes k} \in \widetilde{F}$ for all $\kappa \in S$. Therefore

$$
\begin{aligned}
\kappa^{N} f^{\otimes N}+\kappa^{N-1}\left(f^{\otimes(N-1)}+\pi_{H \odot N}\left(f^{\otimes(N-1)} \otimes V f\right)\right) & \\
+\kappa^{N-2}\left(f^{\otimes(N-2)}+\pi_{H \odot(N-1)}\left(f^{\otimes(N-2)} \otimes V f\right)\right. & \left.+\pi_{H \odot N}\left(f^{\otimes(N-2)} \otimes(V f)^{\otimes 2}\right)\right) \\
& +\cdots+\left(V f+\cdots+(V f)^{\otimes N}\right) \in \widetilde{F} .
\end{aligned}
$$

By considering $\bigoplus_{k=1}^{N} H^{\odot k} / \widetilde{F}$, from the earlier argument, we deduce that 
$f^{\otimes N} \in \widetilde{F}$ and consequently $H^{\odot N} \subset \widetilde{F}$. Moreover,

$$
f^{\otimes(N-1)}+\underbrace{\pi_{H \odot N}\left(f^{\otimes(N-1)} \otimes V f\right)}_{\in H^{\odot N} \subset \widetilde{F}} \in \widetilde{F},
$$

so $f^{\otimes(N-1)} \in \widetilde{F}$. It follows that $H^{\odot(N-1)} \oplus H^{\odot N} \subset \widetilde{F}$ and so for all $1 \leq k \leq N$, $f^{\otimes k} \in \widetilde{F}$. Therefore (13) holds.

To prove that $e^{V} \otimes W$ has a simple spectrum we proceed similarly. Given $N \geq 1$, we denote by $\widetilde{F}_{1}$ the cyclic space generated by $\sum_{k=1}^{N} f^{\otimes k} \otimes g$. Then

$$
\left(\sum_{k=1}^{N}(\kappa f+V f)^{\otimes k}\right) \otimes g \in \widetilde{F}_{1} .
$$

Now repeat the argument used to prove the simplicity of the spectrum of $e^{V}$ (together with the fact that $f^{\otimes k} \otimes g$ is a $V^{\odot k} \otimes W$-cyclic vector for $\left.H^{\odot k} \otimes G\right)$.

Yet one more property can be deduced from the above proof:

LEMma 3. Under the assumptions of Proposition 1 the unitary operator $e^{V} \oplus\left(e^{V} \otimes W\right) \oplus W$ still has a simple spectrum.

Proof. Indeed, all we need to show is that for $k, l \geq 1, \sigma_{V}^{* k} \perp \sigma_{V}^{* l} * \sigma_{W}$, $\sigma_{V}^{* l} * \sigma_{W} \perp \sigma_{W}$ and $\sigma_{V}^{* k} \perp \sigma_{W}$. In all three cases the proof goes along the same lines. In order to obtain $\sigma_{V}^{* k} \perp \sigma_{V}^{* l} * \sigma_{W}$ it is enough to prove that

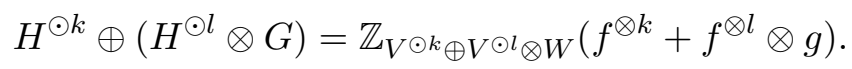

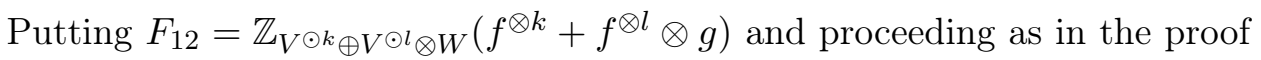
of Proposition 1 we obtain

$$
\begin{aligned}
& \frac{1}{2^{k}}(\kappa f+V f)^{\otimes k}+\frac{1}{2^{l}}(\kappa f+V f)^{\otimes l} \otimes(\kappa g+W g) \in F_{12}, \\
& \frac{1}{2^{k}}(\kappa f+V f)^{\otimes k}+\frac{1}{2^{l}}(\kappa f+V f)^{\otimes l} \otimes(\widetilde{\kappa} g+W g) \in F_{12},
\end{aligned}
$$

so by subtracting, $(\kappa f+V f)^{\otimes l} \otimes g \in F_{12}$. By considering the coefficient of $\kappa^{l}$ knowing that the number of $\kappa \mathrm{s}$ is infinite we find that $f^{\otimes l} \otimes g \in F_{12}$ and therefore $H^{\odot l} \otimes G \subset F_{12}$. Since $f^{\otimes k}+f^{\otimes l} \otimes g \in F_{12}$, also $f^{\otimes k} \in F_{12}$ and (14) follows.

In the remaining cases we show that

$$
\begin{aligned}
H^{\odot k} \oplus G & =F_{13}:=\mathbb{Z}_{V \odot k} \oplus W \\
\left(H^{\odot l} \otimes G\right) \oplus G & \left.=F_{23}:=\mathbb{Z}_{V \odot l}{ }^{\odot k}+g\right), \\
& \left(f^{\otimes l} \otimes g+g\right) .
\end{aligned}
$$

Lemma 4. Assume that $V$ and $W$ are unitary simple spectrum operators on separable Hilbert spaces. Assume moreover that for infinitely many $0<$ 
$\kappa<1$ we have

$$
\begin{aligned}
& V^{n_{t}^{(\kappa)}} \rightarrow \kappa \mathrm{Id}+(1-\kappa) V, \quad W^{n_{t}^{(\kappa)}} \rightarrow \kappa \mathrm{Id}+(1-\kappa) W \\
& V^{m_{t}^{(\kappa)}} \rightarrow \kappa \operatorname{Id}+(1-\kappa) V, \quad W^{m_{t}^{(\kappa)}} \rightarrow \widetilde{\kappa} \operatorname{Id}+(1-\widetilde{\kappa}) W,
\end{aligned}
$$

where $\kappa \neq \widetilde{\kappa}$ and $0<\widetilde{\kappa}<1$. Then the assertions of Proposition 1 and Lemma 3 also hold true.

Proof. Notice that the map $x \mapsto \frac{x}{1-x}$ is $1-1$ on $(0,1),\left(\frac{\kappa}{1-\kappa}\right)^{q} \neq\left(\frac{\widetilde{\kappa}}{1-\widetilde{\kappa}}\right)^{q}$ for all $q \geq 1$, and subspaces are invariant under multiplication by scalars.

In what follows we assume that the spectral measures of the unitary operators considered are continuous. The following lemma is well-known (see for example [7, Chapter 14], and also [16]); we give a proof (in a particular case) just for the reader's convenience. We recall that continuity of the spectral measures allows us to assume that all "hyperplanes" of the form $z_{i}=z_{j}$ are of product measure zero. We then consider a relevant multidimensional torus with all these hyperplanes removed.

LEMMA 5. Assume that $V$ and $W$ are unitary operators with simple continuous spectra. Fix $k \geq 1$. If $V^{\odot k} \otimes W$ has a simple spectrum then the spectral multiplicity of $\bar{V}^{\otimes k} \otimes W$ is uniform and equal to $k$ !.

Proof. By the argument in the proof of Lemma 2 the essential values of $M_{V \otimes k \otimes W}$ are equal (a.e. with respect to the measure $\sigma_{V}^{* k} * \sigma_{W}$ ) either to the number of atoms for conditional measures $\eta_{c}$ which are purely atomic, or to infinity if $\eta_{c}$ has a continuous part: here

$$
\sigma_{V}^{\otimes k} \otimes \sigma_{W}=\int_{\mathbb{T}} \eta_{c} \otimes \delta_{c} d\left(\sigma_{V}^{* k} * \sigma_{W}\right)(c),
$$

where the disintegration is given by the map $S: \mathbb{T}^{k} \times \mathbb{T} \rightarrow \mathbb{T}$ :

$$
\mathbb{T}^{k} \times \mathbb{T} \ni\left(z_{1}, \ldots, z_{k}, w\right) \mapsto z_{1} \cdot \ldots \cdot z_{k} \cdot w \in \mathbb{T}
$$

and we identify $V$ (resp. $W$ ) with multiplication by $z$ on $L^{2}\left(\mathbb{T}, \sigma_{V}\right.$ ) (resp. $\left.L^{2}\left(\mathbb{T}, \sigma_{W}\right)\right)$. We assume that the conditional measures $\eta_{c}$ are purely atomic with finitely many atoms (the remaining cases are treated similarly). By tak$\operatorname{ing}\left(z_{1}, \ldots, z_{k}, w\right)$ with $z_{i} \neq z_{j}$ and considering $T_{\tau}\left(z_{1}, \ldots, z_{k}, w\right)=\left(z_{\tau(1)}, \ldots\right.$, $\left.z_{\tau(k)}, w\right), \tau \in \Sigma_{k}$, we see that $\sigma_{V}^{\otimes k} \otimes \sigma_{W}$ is invariant under $T_{\tau}$, and since $S \circ T_{\tau}$ on the fibres of $S$ does not depend on $\tau \in \Sigma_{k}$ and the disintegration (15) is unique, also the conditional measures $\eta_{c}$ are $T_{\tau}$-invariant. If we choose one element from each set $\left.\left\{z_{\tau(1)}, \ldots, z_{\tau(k)}, w\right): \tau \in \Sigma_{k}\right\}$ so that the resulting subset $A$ of $\mathbb{T}^{k} \times \mathbb{T}$ is measurable, then the subspace of functions with support in $A$ is closed, invariant, and isomorphic to $\operatorname{Sym}\left(L^{2}\left(\mathbb{T}^{k}, \mu^{\otimes k}\right)\right) \otimes L^{2}(\mathbb{T}, \nu)$ (indeed, a symmetric function takes the same values at each point $\left.T_{\tau}\left(z_{1}, \ldots, z_{k}, w\right)\right)$, whence the action is isomorphic to 
$V^{\odot k} \otimes W$. Since the latter automorphism is assumed to have a simple spectrum, given $c \in \mathbb{T}$ (a.a. with respect to the convolution measure) we can have only one atom of $\eta_{c}$ in $S^{-1}(c) \cap A$. It follows that there are $k$ ! atoms for $\eta_{c}$ in $S^{-1} c$, and the result follows.

We now pass to the multiplicity problem for Koopman representations coming from direct products of measure preserving transformations.

Proposition 2. Assume that $T$ and $S$ are ergodic automorphisms of standard probability Borel spaces $(X, \mathcal{B}, \mu)$ and $(Y, \mathcal{C}, \nu)$ respectively. Assume that $V=U_{T}$ and $W=U_{S}$ on the corresponding $L_{0}^{2}$-subspaces satisfy the assumptions of Lemma 4 . Then the spectral multiplicities of $U_{T^{\times k} \times S}$ on the corresponding $L_{0}^{2}$-space are equal to $\{1, k, k(k-1), \ldots, k !\}$.

Proof. By Lemma 3, the subspaces "realizing" $(k-i)$ ! as a multiplicity value for $U_{T^{\times k}} \otimes U_{S}$ on $L_{0}^{2}$ are, up to permutations of coordinates, given by $L_{0}^{2}(X, \mu)^{\otimes(k-i)} \otimes(\mathbb{C} \cdot 1)^{\otimes i} \otimes(\mathbb{C} \cdot 1) \oplus L_{0}^{2}(X, \mu)^{\otimes(k-i)} \otimes(\mathbb{C} \cdot 1)^{\otimes i} \otimes L_{0}^{2}(Y, \nu)$ (indeed, $\left.\sigma_{U_{T}}^{*(k-i)} \perp \sigma_{U_{T}}^{*(k-i)} * \sigma_{U_{S}}\right)$ and there are $\left(\begin{array}{c}k \\ i\end{array}\right)$ such subspaces.

Recall that by the theory of linked approximation [16] (see Section 3 there for basic definitions) the assumptions of Lemma 4 on $V=U_{T}$ are satisfied for a residual set of $T \in \operatorname{Aut}(X, \mathcal{B}, \mu)$. Now, for $\kappa \neq \kappa^{\prime}$ the corresponding subsequences $\left(n_{k}^{(\kappa)}\right)$ and $\left(n_{k}^{\left(\kappa^{\prime}\right)}\right)$ can have only a finite number of common elements. We split $\left(n_{k}^{(\kappa)}\right)$ into two subsequences $\left(n_{k, 1}^{(\kappa)}\right)$ and $\left(n_{k, 2}^{(\kappa)}\right)$ and then we use a general fact that given $\underline{m}=\left(m_{k}\right)$ with $m_{k} \rightarrow \infty$ and $0<\beta<1$ the set of those transformations of $(X, \mathcal{B}, \mu)$ that admit linked approximation along a subsequence of $\underline{m}$ with the proportion of subtowers equal to $\beta$ is still residual. Taking the intersection over all $\kappa \mathrm{s}$ for $\underline{m}=\left(n_{k, j}^{(\kappa)}\right), j=1,2$, we still obtain a residual set.

Corollary 1. There exists a residual set of $T \in \operatorname{Aut}(X, \mathcal{B}, \mu)$ such that for each $T$ in this subset there exists a residual set of $S \in \operatorname{Aut}(X, \mathcal{B}, \mu)$ such that the assertion of Proposition 2 holds.

Recall also that if $T$ is weakly mixing then $\sigma_{U_{T}}$ is continuous (recall that we consider $U_{T}$ on the underlying $L_{0}^{2}$-space), and therefore $e^{U_{T}}$ is unitarily isomorphic to the Koopman operator of the canonical Gaussian system $G_{\sigma_{U_{T}}}$ given by $\sigma_{U_{T}}$ whenever $T$ has a simple spectrum (see e.g. [7]). The proof of Corollary 1 and Lemma 3 now lead directly to the following.

COROllary 2. There exists a residual set of $T \in \operatorname{Aut}(X, \mathcal{B}, \mu)$ such that for each $T$ in this subset there exists a residual set of $S \in \operatorname{Aut}(X, \mathcal{B}, \mu)$ such that the automorphism $G_{\sigma_{U_{T}}} \times S$ has a simple spectrum. 
Notice that for each Gaussian-Kronecker automorphism $G_{\sigma}$ (see e.g. [24]) the operator $V_{\sigma}$ satisfies the assumptions on $V=U_{T}$ in Proposition 1; indeed, each function of modulus at most 1 belongs to the weak closure of $\left\{z^{n}: n \in\right.$ $\mathbb{Z}\}$ in $L^{2}(\mathbb{T}, \sigma)$. It follows that for each Gaussian-Kronecker automorphism $G_{\sigma}, G_{\sigma} \times S$ has a simple spectrum for a residual set of $S \in \operatorname{Aut}(X, \mathcal{B}, \mu)$.

Another class of unitary operators satisfying the assumptions of Lemma 4 can be obtained using infinite measure preserving transformations. Indeed, the easiest way is to repeat linked rank-1 type constructions in the infinite measure case (taking care to obtain infinitely many $\kappa \mathrm{s}$ as proportions of two subtowers in the linked approximation). In this case we consider $U_{T}$ on the whole $L^{2}(X, \mathcal{B}, \mu)$, and the same argument as in the finite measure preserving case shows that there is no set of finite positive measure which is $T$-invariant. It follows that the corresponding Poisson suspension automorphism $\widetilde{T}$ (see e.g. [7], [9], [31]) is weakly mixing and has a simple spectrum. Moreover, for a residual set of $S \in \operatorname{Aut}(X, \mathcal{B}, \mu), \widetilde{T} \times S$ has a simple spectrum.

2. Spectral analysis of $T_{\varphi} \times T_{\varphi}$. Assume that $T:(X, \mathcal{B}, \mu) \rightarrow(X, \mathcal{B}, \mu)$ is an ergodic automorphism of a standard probability Borel space $(X, \mathcal{B}, \mu)$. Recall that $C(T)$ denotes the centralizer of $T$, i.e. the set of transformations of $(X, \mathcal{B}, \mu)$ commuting with $T$. Let $\varphi: X \rightarrow G$ be a cocycle, where $G$ is an Abelian compact metric group with Haar measure $m_{G}$. Define $T_{\varphi}: X \times G \rightarrow$ $X \times G$ by

$$
T_{\varphi}(x, g)=(T x, \varphi(x)+g) \quad \text { for }(x, g) \in X \times G .
$$

It follows that $T_{\varphi}$ is an automorphism of $\left(X \times G, \mathcal{B} \otimes \mathcal{B}(G), \mu \otimes m_{G}\right)$. For each $n \in \mathbb{Z}$ we have $\left(T_{\varphi}\right)^{n}(x, g)=\left(T^{n} x, \varphi^{(n)}(x)+g\right)$, where

$$
\varphi^{(n)}(x)= \begin{cases}\varphi(x)+\varphi(T x)+\cdots+\varphi\left(T^{n-1} x\right) & \text { if } n>0, \\ 0 & \text { if } n=0, \\ -\left(\varphi\left(T^{n} x\right)+\cdots+\varphi\left(T^{-1} x\right)\right) & \text { if } n<0 .\end{cases}
$$

A cocycle $\varphi$ is called a coboundary if there is a measurable function $j: X \rightarrow G$ (called a transfer function) such that $\varphi=j-j \circ T$ (we also write $\varphi=\operatorname{cob}(T)$ ); in fact, by ergodicity of $T, j$ is unique up to an additive constant.

Given $\chi \in \widehat{G}$ we define

$$
V_{\varphi, T}^{\chi}: L^{2}(X, \mu) \rightarrow L^{2}(X, \mu), \quad V_{\varphi, T}^{\chi} f=\chi \circ \varphi \cdot f \circ T .
$$

Then $V_{\varphi, T}^{\chi}$ is isomorphic to $U_{T_{\varphi}}$ acting on the subspace $L^{2}(X, \mu) \otimes \chi$ considered as a subspace of $L^{2}\left(X \times G, \mu \otimes m_{G}\right)$ and (up to isomorphism)

$$
U_{T_{\varphi}}=\bigoplus_{\chi \in \widehat{G}} V_{\varphi, T}^{\chi}
$$

Notice also that $V_{\varphi, T}^{1}=U_{T}$. 
From now on we will assume that $T$ is weakly mixing. Notice that $T_{\varphi} \times T_{\varphi}$ is naturally identified with $(T \times T)_{\varphi \times \varphi}$. Therefore, it follows from (16) that (up to natural identifications)

$$
U_{T_{\varphi} \times T_{\varphi}}=U_{T_{\varphi}} \otimes U_{T_{\varphi}}=\bigoplus_{(\chi, \eta) \in \widehat{G} \times \widehat{G}} V_{\varphi, T}^{\chi} \otimes V_{\varphi, T}^{\eta} .
$$

Denote by $\sigma_{\chi}$ the maximal spectral type of $V_{\varphi, T}^{\chi}$.

Proposition 3. Assume that

(a) $V_{\varphi, T}^{\chi}$ has a simple spectrum for each $\chi \in \widehat{G}$;

(b) $V_{\varphi, T}^{\chi} \otimes V_{\varphi, T}^{\eta}$ has a simple spectrum for each $(\chi, \eta) \in \widehat{G} \times \widehat{G}$ whenever $\chi \neq \eta$

(c) $V_{\varphi, T}^{\chi} \otimes V_{\varphi, T}^{\chi}$ has uniform multiplicity 2 for each $\chi \in \widehat{G}$;

(d) $\sigma_{\chi} * \sigma_{\eta} \perp \sigma_{\chi^{\prime}} * \sigma_{\eta^{\prime}}$ whenever the sets $\{\chi, \eta\}$ and $\left\{\chi^{\prime}, \eta^{\prime}\right\}$ are different. Then $U_{T_{\varphi} \times T_{\varphi}}$ has uniform multiplicity 2 on the corresponding $L_{0}^{2}$-space.

Proof. The result follows directly from (17) rewritten as

$$
U_{T_{\varphi} \times T_{\varphi}}=\bigoplus_{\chi \in \widehat{G}}\left(V_{\varphi, T}^{\chi} \otimes V_{\varphi, T}^{\chi}\right) \oplus \bigoplus_{\{\chi, \eta\} \subset \widehat{G} ; \chi \neq \eta}\left(\left(V_{\varphi, T}^{\chi} \otimes V_{\varphi, T}^{\eta}\right) \oplus\left(V_{\varphi, T}^{\eta} \otimes V_{\varphi, T}^{\chi}\right)\right) .
$$

Lemma 6. Assume that for each $\chi \in \widehat{G}, V_{\varphi, T}^{\chi}$ has a simple spectrum, and for each pair $(\chi, \eta) \in \widehat{G} \times \widehat{G}$ with $\chi \neq \eta$ there exist subsequences $\left(n_{t}\right)$, $\left(m_{t}\right)$ such that

(a) $\left(V_{\varphi, T}^{\chi}\right)^{n_{t}} \rightarrow \frac{1}{2}\left(\mathrm{Id}+V_{\varphi, T}^{\chi}\right)$ and $\left(V_{\varphi, T}^{\eta}\right)^{n_{t}} \rightarrow \frac{1}{2}\left(\mathrm{Id}+V_{\varphi, T}^{\eta}\right)$;

(b) $\left(V_{\varphi, T}^{\chi}\right)^{m_{t}} \rightarrow \frac{1}{2}\left(\mathrm{Id}+V_{\varphi, T}^{\chi}\right)$ and $\left(V_{\varphi, T}^{\eta}\right)^{m_{t}} \rightarrow \frac{1}{2}\left(\mathrm{Id}+c V_{\varphi, T}^{\eta}\right)$ with $c=$ $c(\chi, \eta) \neq 1$.

Assume moreover that for $\{\chi, \eta\} \neq\left\{\chi^{\prime}, \eta^{\prime}\right\}$ there exists a subsequence $\left(p_{t}\right)$ such that

(c) $\left(V_{\varphi, T}^{\gamma}\right)^{p_{t}} \rightarrow d_{\gamma} \cdot$ Id for $\gamma \in\left\{\chi, \eta, \chi^{\prime}, \eta^{\prime}\right\}$ with $\left|d_{\gamma}\right|=1$ and

$$
d_{\chi} \cdot d_{\eta} \neq d_{\chi^{\prime}} \cdot d_{\eta^{\prime}}
$$

Then the assertion of Proposition 3 holds.

Proof. By (a), (b) and Lemma 1 the spectrum of $V_{\varphi, T}^{\chi} \otimes V_{\varphi, T}^{\eta}$ is simple for $\chi \neq \eta$. By (a) and Lemma $2, V_{\varphi, T}^{\chi}$ has uniform multiplicity 2. In view of (c), $\left(V_{\varphi, T}^{\chi} \otimes V_{\varphi, T}^{\eta}\right)^{p_{t}} \rightarrow d_{\chi} \cdot d_{\eta} \cdot$ Id. Recall (see [17]) that if $V$ and $W$ are unitary operators on separable Hilbert spaces and if $V^{n_{t}} \rightarrow c \cdot \mathrm{Id}$ and $W^{n_{t}} \rightarrow d \cdot \mathrm{Id}$ with $c \neq d,|c|=|d|=1$, then $\sigma_{V} \perp \sigma_{W}$. This yields mutual disjointness of the spectral measures of the operators $V_{\varphi, T}^{\chi} \otimes V_{\varphi, T}^{\eta}$. 
REMARK 1. Notice that the weak convergences above exclude any point spectrum of $U_{T_{\varphi}}$ (in the orthocomplement of constants). In other words, $T_{\varphi}$ is still weakly mixing.

3. Rigid rank 1 automorphisms admitting linked ( $n, n+1)$-approximation. We will consider automorphisms $T$ that are simultaneously approximated by two kind of towers:

(B) $\left(B_{t}, T B_{t}, \ldots, T^{r_{t}-1} B_{t}\right)$, where "practically" (that is, with the precise speed being a function of $r_{t}$ ) $T^{r_{t}} B_{t}=B_{t}$; these are called rigid towers;

(C) $\left(C_{t}, T C_{t}, \ldots, T^{n_{t}-1} C_{t}\right)$, where $C_{t}=C_{t}^{1} \cup C_{t}^{2}$, both $C_{t}^{i}$ are of the same measure and "practically" (that is, with the precise speed being a function of $\left.n_{t}\right) T^{n_{t}} C_{t}^{1}=C_{t}^{1}$ while $\left(C_{t}^{2}, T C_{t}^{2}, \ldots, T^{n_{t}-1} C_{t}^{2}, T^{n_{t}} C_{t}^{2}\right)$ is still a tower and with the same meaning of the equality $T^{n_{t}+1} C_{t}^{2}=$ $C_{t}^{2}$ as before; such a tower is called a linked tower.

Both sequences of towers $\left(B_{t}, T B_{t}, \ldots, T^{r_{t}-1} B_{t}\right)_{t},\left(C_{t}, T C_{t}, \ldots, T^{n_{t}-1} C_{t}\right)_{t}$ are assumed to generate the Borel structure of the underlying space of $T$.

It follows from [16] that a typical automorphism admits such a simultaneous approximation. Therefore it can be assumed additionally that $T$ is weakly mixing.

In what follows we will tacitly (although without restricting generality) assume that the sequences as above are nested: by that we mean not only that the $(B)$ - and $(C)$-towers are nested but also that they refine each other (see [15] for details).

In the case of approximation by nested $C$-towers one speaks about linked approximation of type $\left(n_{t}, n_{t}+1\right)$.

Notice that (B) implies

$$
U_{T^{r_{t}}} \rightarrow \mathrm{Id}
$$

while $(\mathrm{C})$ implies that

$$
U_{T^{n_{t}+1}} \rightarrow \frac{1}{2}\left(\mathrm{Id}+U_{T}\right) .
$$

We should notice, however, that neither (18) nor (19) requires so strong assumptions as (B) and (C) respectively. For example, once for some $m \geq 2$, $T$ admits a good approximation by towers

$$
\left(C_{t}^{(j)}, T C_{t}^{(j)}, \ldots T^{n_{t}-1} C_{t}^{(j)}\right)
$$

for $j=1, \ldots, m$, each separate tower being of type (C), then (19) holds true. We will refer to such a situation as linked approximation by $m$ towers of the same height.

We can drop the assumption that in the partition $C_{t}=C_{t}^{1} \cup C_{t}^{2}$, both $C_{t}^{i}$ are of the same measure, just assuming a freely chosen proportion $0<\beta<1$. 
This will lead to linked approximation of type $\left(n_{t}, n_{t}+1\right)$ with proportion $\beta$ and in particular we will have $U_{T^{n+1}} \rightarrow \beta \mathrm{Id}+(1-\beta) U_{T}$.

3.1. Lifting weak convergences by cocycles. We will now define certain cocycles with values in the group $G$ for $T$ satisfying the above assumptions (B) and (C). The construction will satisfy the following general scheme. When we arrive at a rigid tower we assume that $\varphi$ is defined on all levels except for a few, including the top level, and $\varphi$ is constant on each level $T^{i} B_{t}$ for those $0 \leq i \leq r_{t}-2$ on which $\varphi$ is already defined. We first put arbitrary constant values of $\varphi$ on the levels $T^{i} B_{t}$ for $0 \leq i \leq r_{t}-2$ on which $\varphi$ is not defined yet. Next, such a tower is divided into many subcolumns, the top level of each (except for the last one) is sent by $T$ to the bottom level of the next ("visually" right) subcolumn. We then put constant values of $\varphi$ on the tops of all subcolumns, keeping the top of the last subcolumn undefined. The situation is similar when we arrive at a linked tower - we assume that $\varphi$ is defined on all levels (taking constant values on such levels) except for some levels including the level $T^{n_{t}-1} C_{t}$. We first put arbitrary constant values of $\varphi$ on $T^{i} C_{t}\left(0 \leq i \leq n_{t}-2\right)$ where $\varphi$ is not defined yet. Then such a tower is divided into subcolumns which refine also the subtowers based on $C_{t}^{1}$ and $C_{t}^{2}$ respectively. We put constant values of $\varphi$ on all top levels of the subcolumns contained in the tower with bottom $C_{t}^{1}$, while for subcolumns contained in the other subtower we put constant values of $\varphi$ on the top level and its image via $T$-we keep $\varphi$ undefined only on the image via $T$ of the top of the last subcolumn.

We will have a COUNTABLE list of "parameters" which control the behaviour of extended automorphisms along different subsequences, meaning that for each "parameter" we "reserve" a subsequence (of $\left(n_{t}\right)$ or $\left(r_{t}\right)$ ) and we put the values of $\varphi$ so that we have a special behaviour of the extended automorphism along this subsequence.

As the first example of the mechanism described above we require that if $G=\mathbb{Z}_{m}$ (the cyclic group of order $m$ ) then for a subsequence $\left(r_{k}^{(1)}\right)$ of $\left(r_{t}\right)$ the cocycle $\varphi$ is defined in such a way that

$$
\varphi^{\left(r_{k}^{(1)}\right)}(x)=1
$$

for all $x$ in the union of all levels of the tower except for the last subcolumn. Notice that then $B_{t_{k}} \times\{0\}\left(r_{k}^{(1)}=r_{t_{k}}\right)$ is the base of a rigid tower of height $m \cdot n_{t_{k}}$ for $T_{\varphi}$.

Rigid towers are also used to obtain the following properties:

- simple spectrum of $V_{\varphi, T}^{\chi}$ (which is classical);

- property (c) from Lemma 6. 
More precisely, in our construction we will have the following: given $\chi \in \widehat{G}$ there exists a subsequence $\left(r_{k}^{(\chi)}\right)$ of $\left(r_{t}\right)$ such that $\chi\left(\varphi^{\left(r_{k}^{(\chi)}\right)}\right) \rightarrow d_{\chi}$ in measure, where $\left|d_{\chi}\right|=1$. Then

$$
\left(V_{\varphi, T}^{\chi}\right)^{r_{k}^{(\chi)}} \rightarrow d_{\chi} \cdot \text { Id } \quad \text { whenever } \quad \chi\left(\varphi^{\left(r_{k}^{(\chi)}\right)}\right) \rightarrow d_{\chi} .
$$

Indeed, to prove $(21)$ it is enough to consider a bounded $f \in L^{2}(X, \mu)$ which is constant on the levels of the rigid towers $\left(f=f_{i}\right.$ on $\left.T^{i} B_{t_{k}}\right)$. We then have

$$
\begin{aligned}
\left\langle\left(V_{\varphi, T}^{\chi}\right)^{r_{k}^{(\chi)}} f, f\right\rangle & =\sum_{i=0}^{r_{t_{k}}-1} \int_{T^{i} B_{t_{k}}}\left(\chi\left(\varphi^{\left(r_{t}^{(\chi)}\right)}\right) \pm d_{\chi}\right)\left|f_{i}\right|^{2} d \mu+\mathrm{o}(1) \\
& =d_{\chi} \sum_{i=0}^{r_{t_{k}}-1}\left|f_{i}\right|^{2}+\int_{X}\left(\chi\left(\varphi^{\left(r_{t}^{(\chi)}\right)}\right)-d_{\chi}\right)|f|^{2} d \mu+\mathrm{o}(1) \rightarrow d_{\chi}\langle f, f\rangle .
\end{aligned}
$$

We use linked towers to obtain properties (a) and (b) of Lemma 6. More precisely, fix $g, h \in G$ and a subsequence $\left(n_{k}^{(g, h)}\right)$ of $\left(n_{t}\right)$. We put the values of $\varphi$ in such a way that

$$
\begin{gathered}
\varphi^{\left(n_{k}^{(g, h)}\right)}(x)=g \quad \text { for } x \in \bigcup_{i=0}^{n_{t_{k}}-1} T^{i} C_{t_{k}}^{1}, \\
\varphi^{\left(n_{k}^{(g, h)}+1\right)}(x)=h \quad \text { for } x \in \bigcup_{i=0}^{n_{t_{k}}} T^{i} C_{t_{k}}^{2} \text { except in the last subcolumn. }
\end{gathered}
$$

LEMMA 7. Under the above assumptions,

$$
\left(V_{\varphi, T}^{\chi}\right)^{n_{k}^{(g, h)}+1} \rightarrow \frac{1}{2}\left(\chi(h) \cdot \operatorname{Id}+\chi(g) \cdot V_{\varphi, T}^{\chi}\right) .
$$

Proof. Again we take a bounded function which is constant on the levels of the corresponding linked towers $\left(f=f_{i}\right.$ on $\left.T^{i} C_{t_{k}}\right)$. Define $\widetilde{C}_{1}=$ $\bigcup_{i=0}^{n_{t_{k}}-1} T^{i} C_{t_{k}}^{1}$ and $\widetilde{C}_{2}=\bigcup_{i=0}^{n_{t_{k}}} T^{i} C_{t_{k}}^{2}$, where $n_{t_{k}}=n_{k}^{(g, h)}$. We have

$$
\begin{aligned}
\left\langle\left(V_{\varphi, T}^{\chi}\right)^{n_{k}^{(g, h)}+1} f, f\right\rangle= & \int_{\widetilde{C}_{1}} \chi\left(\varphi^{\left(n_{t_{k}}\right)}(x)\right) \chi\left(\varphi\left(T^{n_{t_{k}}} x\right)\right) f \circ T\left(T^{n_{t_{k}}} x\right) \overline{f(x)} d \mu(x) \\
& +\int_{\widetilde{C}_{2}} \chi\left(\varphi^{\left(n_{t_{k}}+1\right)}(x)\right) f\left(T^{n_{t_{k}}+1} x\right) \overline{f(x)} d \mu(x)+\mathrm{o}(1) \\
= & \sum_{i=0}^{n_{t_{k}}-1} \int_{T^{i} C_{t_{k}}^{1}} \chi(g) \chi(\varphi(x)) f_{i+1} \bar{f}_{i} d \mu(x) \\
& +\sum_{i=0}^{n_{t_{k}}} \int_{T^{i} C_{t_{k}}^{2}} \chi(h) f_{i} \bar{f}_{i} d \mu(x)+\mathrm{o}(1)+\mathrm{o}(1)
\end{aligned}
$$




$$
\begin{aligned}
= & \chi(g) \int_{\widetilde{C}_{1}} \chi(\varphi(x)) f(T x) \overline{f(x)} d \mu(x) \\
& +\chi(h) \int_{\widetilde{C}_{2}} f(x) \overline{f(x)} d \mu(x)+\mathrm{o}(1)+\mathrm{o}(1)+\mathrm{o}(1) \\
\rightarrow & \chi(g) \cdot \frac{1}{2} \int_{X} \chi(\varphi(x)) f(T x) \overline{f(x)} d \mu(x)+\chi(h) \cdot \frac{1}{2} \int_{X} f(x) \overline{f(x)} d \mu(x),
\end{aligned}
$$

where the last equality follows from the fact that both $f$ and $\varphi$ are constant on the levels $T^{i} C_{t_{k}}$. Now (24) follows.

The above lemma (which will not be used in what follows exactly as formulated) is about a lift of weak convergence of some powers of $U_{T}$ on selected subspaces and not on the whole $L^{2}$-space. However, in the lemma below we will see that we may speak about global convergence of some powers of $U_{T_{\varphi}}$ to some "good" operator; see also Appendix about lifting weak convergence for so called Rokhlin extensions.

Lemma 8. Assume that $G=\mathbb{Z}_{m}$ and that a subsequence $\left(n_{k}^{(0)}\right)$ of $\left(n_{t}\right)$ satisfies

$$
\varphi^{\left(n_{k}^{(0)}\right)}(x)=0 \quad \text { for } x \in \bigcup_{i=0}^{n_{t_{k}}-1} T^{i} C_{t_{k}}^{1}
$$

and

$$
\varphi^{\left(n_{k}^{(0)}+1\right)}(x)=0 \quad \text { for } x \in \bigcup_{i=0}^{n_{t_{k}}} T^{i} C_{t_{k}}^{2} \text { except in the last subcolumn. }
$$

Then $T_{\varphi}$ admits linked approximation by $m$ towers of the same height and in particular

$$
U_{T_{\varphi}}^{n_{k}^{(0)}+1} \rightarrow \frac{1}{2}\left(\mathrm{Id}+U_{T_{\varphi}}\right) .
$$

Moreover, there exists a subsequence $\left(r_{k}^{(0)}\right)$ of $\left(r_{t}\right)$ such that $\varphi^{\left(r_{k}^{(0)}\right)}=0$ on the whole tower except the last column. This leads to a sequence of $m$ towers of heights $r_{k}^{(0)}$, and the sequence $\left(r_{k}^{(0)}\right)$ is a rigidity sequence for $T_{\varphi}$.

Proof. It remains to notice that for each $g \in \mathbb{Z}_{m}, C_{t_{k}} \times\{g\}$ is the base of a linked tower: two rigid subtowers of heights $n_{t_{k}}$ and $n_{t_{k}}+1$ respectively are obtained by taking $C_{t_{k}}^{j} \times\{g\}, j=1,2$, as bases.

4. Spectral analysis of double extensions of $T$. The spectral analysis of a double extension of $T$ given below is similar to Robinson's analysis in [29] and [30]. 
Let $\mathbb{A}$ be an Abelian compact metric group with Haar measure $m_{\mathbb{A}}$. Assume that $v: \mathbb{A} \rightarrow \mathbb{A}$ is a continuous group automorphism. Denote by $\widehat{v}$ the dual automorphism acting on $\widehat{\mathbb{A}}$. We assume that for some $m \geq 2, \widehat{v}^{m}=\mathrm{Id}$ (we take the smallest possible $m$ with this property) and then fix $G=\mathbb{Z}_{m}$. Then a cocycle $\varphi: X \rightarrow \mathbb{Z}_{m}$ is chosen so that all lemmas of the previous section are satisfied. Define a cocycle (for $T_{\varphi}$ ) $\Theta: X \times \mathbb{Z}_{m} \rightarrow \mathbb{A}$ by putting

$$
\Theta(x, i)=v^{i}(\psi(x)),
$$

where $\psi: X \rightarrow \mathbb{A}$ is measurable.

The function $\psi$ will be defined in a while according to the same general rules applied to define $\varphi$.

Put also $S(x, i)=(x, i+1)$. Then clearly

$$
S \in C\left(T_{\varphi}\right)
$$

and also

$$
\Theta \circ S-v \circ \Theta=\operatorname{cob}\left(T_{\varphi}\right)
$$

in fact the transfer function for the coboundary above is simply equal to zero: indeed,

$$
v^{i+1}(a)=v\left(v^{i}(a)\right) \quad \text { for each } i \in \mathbb{Z}_{m}
$$

since $v\left(v^{m-1}\right)=$ Id. If we put $\widetilde{S}(x, i, a)=(x, i+1, v(a))$ then it follows from (25) and (26) that

$$
\widetilde{S} \in C\left(T_{\varphi}\right) .
$$

Therefore the following lemma results from [29] or [12].

Lemma 9. For each $\xi \in \widehat{\mathbb{A}}$ the unitary operators $V_{\Theta, T_{\varphi}}^{\xi}$ and $V_{\Theta, T_{\varphi}}^{\widehat{v}(\xi)}$ are isomorphic.

Lemma 10. Assume that $T_{\varphi}$ is ergodic. Let $\left(n_{k}^{(0)}\right)$ be a subsequence as in Lemma 8. Then we can define $\psi$ so that for every $a, b \in \mathbb{A}$ there exists a subsequence $\left(n_{s}^{(0, a, b)}\right)$ of $\left(n_{k}^{(0)}\right)$ such that for each $\xi \in \widehat{\mathbb{A}}$ we have

$$
\left(V_{\Theta, T_{\varphi}}^{\xi}\right)^{n_{s}^{(0, a, b)}} \rightarrow \frac{1}{2}\left(\frac{1}{m} \sum_{j=0}^{m-1} \xi\left(v^{j} b\right) \cdot \operatorname{Id}+\frac{1}{m} \sum_{j=0}^{m-1} \xi\left(v^{j} a\right) \cdot V_{\Theta, T_{\varphi}}^{\xi}\right) .
$$

Proof. Given $n \geq 1$ put

$$
W_{n}(x)=\psi(x)+v^{\varphi(x)}(\psi(T x))+\cdots+v^{\varphi^{(n-1)}(x)}\left(\psi\left(T^{n-1} x\right)\right) .
$$

Notice that $\Theta^{(n)}(x, i)=v^{i}\left(W_{n}(x)\right)$. We claim that

$$
\begin{aligned}
& v^{\varphi(x)}\left(W_{n}(T x)\right)=W_{n}(x) \quad \text { provided that } \\
& (*) \varphi^{(n)}(x)=0, \psi(x)=\psi\left(T^{n} x\right) .
\end{aligned}
$$


Indeed, we have

$$
\begin{aligned}
v^{-\varphi(x)} W_{n}(x)= & v^{-\varphi(x)}(\psi(x))+\psi(T x)+v^{\varphi(T x)}\left(\psi\left(T^{2} x\right)\right) \\
& +\cdots+v^{\varphi^{(n-2)}(T x)}\left(\psi\left(T^{n-1} x\right)\right) \\
= & v^{\varphi^{(n-1)}(T x)}\left(\psi\left(T^{n-1}(T x)\right)\right)+\psi(T x)+v^{\varphi(T x)}(\psi(T(T x))) \\
& +\cdots+v^{\varphi^{(n-2)}(T x)}\left(\psi\left(T^{n-2}(T x)\right)\right)=W_{n}(T x)
\end{aligned}
$$

and (29) follows. Notice that also

$$
v^{\varphi^{(j)}(x)}\left(W_{n}\left(T^{j} x\right)\right)=W_{n}(x)
$$

for $1 \leq j \leq n-1$ once we know that $(*)$ in (29) is satisfied for $x, T x, \ldots$, $T^{j-1} x$.

For all $F, G \in L^{2}\left(X \times \mathbb{Z}_{m}, \mu \otimes m_{\mathbb{Z}_{m}}\right), n \in \mathbb{Z}$ and $\xi \in \widehat{\mathbb{A}}$ we have

$$
\begin{aligned}
\left\langle\left(V_{\Theta, T_{\varphi}}^{\xi}\right)^{n} F, G\right\rangle= & \int_{X \times \mathbb{Z}_{m}} \xi\left(v^{y}(\psi(x))+v^{\varphi(x)+y}(\psi(T x))+\cdots\right. \\
& \left.+v^{\varphi(n-1)}(x)+y\left(\psi\left(T^{n-1} x\right)\right)\right) F\left(\left(T_{\varphi}\right)^{n}(x, y)\right) \overline{G(x, y)} d \mu d y \\
= & \int_{X \times \mathbb{Z}_{m}} \xi \circ v^{y}\left(W_{n}(x)\right) F\left(\left(T_{\varphi}\right)^{n}(x, y)\right) \overline{G(x, y)} d \mu d y .
\end{aligned}
$$

We will now define $\psi$ according to the general scheme with extra claims that

$$
\begin{aligned}
W_{n_{t_{k_{s}}}}(x)=a & \text { for } x \in C_{n_{t_{k_{s}}}}^{1}, \\
W_{n_{t_{k_{s}}}+1}(x)=b & \text { for } x \in C_{n_{t_{k_{s}}}}^{2},
\end{aligned}
$$

where $n_{t_{k_{s}}}=n_{s}^{(0, a, b)}$. Let us first argue that such a requirement on $\psi$ is compatible with the scheme. Indeed, for $x \in C_{n_{t_{k_{s}}}}^{1}$ we have

$$
W_{n_{t_{k_{s}}}}(x)=\psi(x)+v^{\varphi(x)}(\psi(T x))+\cdots+v^{\varphi^{\left(n_{t_{k_{s}}}-1\right)}(x)}\left(\psi\left(T^{n_{t_{k_{s}}}-1} x\right)\right),
$$

where only the last summand is not defined yet, while all others are already defined (and are constant on the corresponding levels). Now, $v^{\varphi^{\left({ }^{(} t_{k_{s}}-1\right)}(x)}$ is an automorphism of $\mathbb{A}$ and we let $\psi\left(T^{n_{t_{k}}}{ }^{-1} x\right)$ be the unique value such that (30) is satisfied. The same reasoning remains valid for the other subtower.

Put $n_{s}^{\prime}=n_{t_{k_{s}}}$ and recall that $T_{\varphi}$ admits a linked approximation of type $\left(n_{s}^{\prime}, n_{s}^{\prime}+1\right)$ by $m$ towers. Fix $F, G \in L^{2}\left(X \times \mathbb{Z}_{m}, \mu \otimes m_{\mathbb{Z}_{m}}\right)$. We will assume that $F$ and $G$ are bounded and constant on the levels of the $m$ towers (the constant values will be denoted $F_{i, j}$ and $G_{i, j}$ respectively). Then we have 
the following sequence of equalities:

$$
\begin{aligned}
\left\langle\left(V_{\Theta, T_{\varphi}}^{\xi}\right)^{n_{s}^{\prime}+1} F, G\right\rangle & \sum_{i=0}^{m-1}\left(\int_{\bigcup_{j=0}^{n_{s}^{\prime}-1}\left(T_{\varphi}\right)^{j}\left(C_{n_{s}^{\prime}}^{1} \times\{i\}\right)} \xi\left(\Theta^{\left(n_{s}^{\prime}+1\right)}\right) \cdot F \circ\left(T_{\varphi}\right)^{n_{s}^{\prime}+1} \cdot \bar{G} d \mu d y\right. \\
& \left.+\int_{\bigcup_{j=0}^{n_{s}^{\prime}}\left(T_{\varphi}\right)^{j}\left(C_{n_{s}^{\prime}}^{2} \times\{i\}\right)} \xi\left(\Theta^{\left(n_{s}^{\prime}+1\right)}\right) \cdot F \circ\left(T_{\varphi}\right)^{n_{s}^{\prime}+1} \cdot \bar{G} d \mu d y\right)+\mathrm{o}(1)
\end{aligned}
$$

and (modulo o(1))

$$
\begin{aligned}
& \sum_{i=0}^{m-1} \int_{\bigcup_{j=0}^{n_{s}^{\prime}}\left(T_{\varphi}\right)^{j}\left(C_{n_{s}^{\prime}}^{2} \times\{i\}\right)} \xi\left(\Theta^{\left(n_{s}^{\prime}+1\right)}\right) \cdot F \circ\left(T_{\varphi}\right)^{n_{s}^{\prime}+1} \cdot \bar{G} d \mu d y \\
& =\sum_{i=0}^{m-1} \sum_{j=0}^{n_{s}^{\prime}} \int_{X \times \mathbb{Z}_{m}} \xi\left(\Theta^{\left(n_{s}^{\prime}+1\right)}\right) \cdot F \circ\left(T_{\varphi}\right)^{n_{s}^{\prime}+1} \cdot \bar{G} \cdot 1_{\left(T_{\varphi}\right)^{j}\left(C_{n_{s}^{\prime}}^{2} \times\{i\}\right)} d \mu d y \\
& =\sum_{i=0}^{m-1} \sum_{j=0}^{n_{s}^{\prime}} \int_{X \times \mathbb{Z}_{m}} \xi\left(\Theta^{\left(n_{s}^{\prime}+1\right)}\right) \cdot F \circ\left(T_{\varphi}\right)^{n_{s}^{\prime}+1} \cdot \bar{G} \cdot 1_{C_{n_{s}^{\prime}}^{2} \times\{i\}} \circ\left(T_{\varphi}\right)^{-j} d \mu d y \\
& +\sum_{i=0}^{m-1} \sum_{j=0}^{n_{s}^{\prime}} \int_{C_{n_{s}^{\prime}}^{2} \times\{i\}} \xi v^{y+\varphi^{(j)}(x)}\left(W_{n_{s}^{\prime}+1}\left(T^{j} x\right)\right) F\left(\left(T_{\varphi}\right)^{n_{s}^{\prime}+1+j}(x, y)\right) \\
& \times \overline{G\left(\left(T_{\varphi}\right)^{j}(x, y)\right)} d \mu(x) d y \\
& =\sum_{i=0}^{m-1} \sum_{j=0}^{n_{s}^{\prime}} \int_{C_{n_{s}^{\prime}}^{2} \times\{i\}} \xi v^{y}\left(W_{n_{s}^{\prime}+1}(x)\right) F\left(\left(T_{\varphi}\right)^{n_{s}^{\prime}+1+j}(x, y)\right) \overline{G\left(\left(T_{\varphi}\right)^{j}(x, y)\right)} d \mu(x) d y \\
& =\sum_{i=0}^{m-1} \sum_{j=0}^{n_{s}^{\prime}} \frac{1}{m} \int_{C_{n_{s}^{\prime}}^{2}} \xi v^{i}\left(W_{n_{s}^{\prime}+1}(x)\right) F\left(\left(T_{\varphi}\right)^{n_{s}^{\prime}+1+j}(x, y)\right) \overline{G\left(\left(T_{\varphi}\right)^{j}(x, y)\right)} d \mu(x)
\end{aligned}
$$

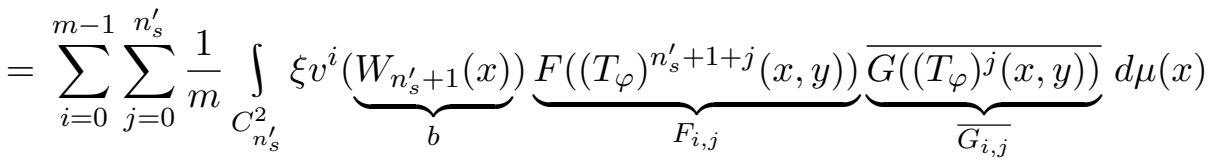

$$
\begin{aligned}
& =\sum_{i=0}^{m-1} \sum_{j=0}^{n_{s}^{\prime}} \frac{1}{m} \int_{C_{n_{s}^{\prime}}} \xi v^{i}(b) F_{i, j} \overline{G_{i, j}} d \mu=\sum_{i=0}^{m-1} \xi v^{i}(b) \frac{1}{2 m n_{s}^{\prime}} \sum_{j=0}^{n_{s}^{\prime}} F_{i, j} \overline{G_{i, j}} \\
& =\sum_{i=0}^{m-1} \xi v^{i}(b) \int_{\bigcup_{j=0}^{n_{s}^{\prime}}\left(T_{\varphi}\right)^{j}\left(C_{n_{s}^{\prime}}^{2} \times\{i\}\right)} F \cdot \bar{G} d \mu .
\end{aligned}
$$


However, $T_{\varphi}$ is ergodic and the sets $\bigcup_{j=0}^{n_{s}^{\prime}}\left(T_{\varphi}\right)^{j}\left(C_{n_{s}^{\prime}}^{2} \times\{i\}\right)$ are almost $T_{\varphi^{-}}$ invariant, and the limit of their measures is exactly $1 / m$. Hence (see e.g. [13, proof of Lemma 2.2])

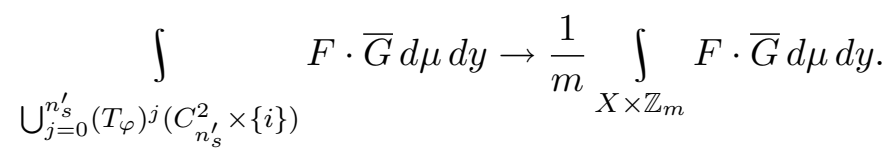

Thus

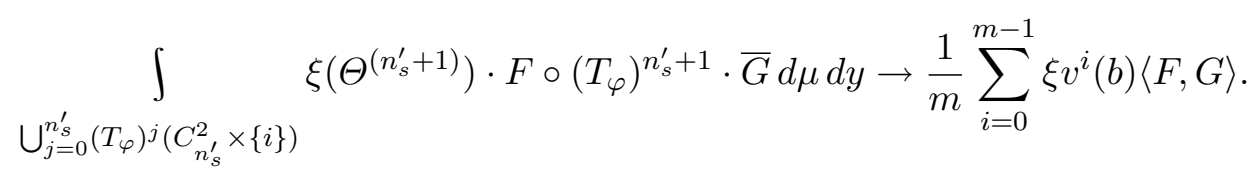

For the remaining summand we notice that

$$
\begin{aligned}
& \sum_{i=0}^{m-1} \int_{\bigcup_{j=0}^{n_{s}^{\prime}-1}\left(T_{\varphi}\right)^{j}\left(C_{n_{s}^{\prime}}^{1} \times\{i\}\right)} \xi\left(\Theta^{\left(n_{s}^{\prime}+1\right)}\right) \cdot F \circ\left(T_{\varphi}\right)^{n_{s}^{\prime}+1} \cdot \bar{G} d \mu d y \\
& \quad=\sum_{i=0}^{m-1} \int_{\substack{\bigcup_{j=0}^{n_{s}^{\prime}-1}\left(T_{\varphi}\right)^{j}\left(C_{n_{s}^{\prime}}^{1} \times\{i\}\right)\\
}\left(\Theta^{n_{s}^{\prime}}\right) \cdot\left(V_{\Theta, T_{\varphi}}^{\xi}(F)\right) \circ\left(T_{\varphi}\right)^{n_{s}^{\prime}} \cdot \bar{G} d \mu d y}
\end{aligned}
$$

and we can repeat the same arguments as for the previous calculation. This completes the proof of the lemma.

Lemma 11. Assume that $T_{\varphi}$ is ergodic. Let $\left(r_{k}^{(0)}\right)$ be the subsequence of Lemma 8. Then we can define $\psi$ so that for each $a \in \mathbb{A}$ there exists a subsequence $\left(r_{l}^{(0, a)}\right)$ of $\left(r_{k}^{(0)}\right)$ such that

$$
\left(V_{\Theta, T_{\varphi}}^{\xi}\right)^{r_{l}^{(0, a)}} \rightarrow \frac{1}{m} \sum_{i=0}^{m-1} \xi\left(v^{i} a\right) \cdot \mathrm{Id} .
$$

Proof. This is a simplified version of the same reasoning as in the proof of the previous lemma.

Given $\xi \in \widehat{\mathbb{A}}$ put

$$
j_{\xi}(a)=\frac{1}{m} \sum_{i=0}^{m-1} \widehat{v}^{i}(\xi)(a), \quad a \in \mathbb{A} .
$$

Two characters $\xi, \zeta \in \widehat{\mathbb{A}}$ are said to be $v$-equivalent (and we write $\xi \equiv \zeta$ ) if $\zeta$ is in the $\widehat{v}$-orbit of $\xi$. We have

(i) $j_{\xi}(0)=1$,

(ii) if $\xi \not \equiv \zeta$ then $j_{\xi} \perp j_{\zeta}$, i.e. $\int_{\mathbb{A}} j_{\xi} \overline{j_{\zeta}} d m_{\mathbb{A}}=0$. 
In particular, for nonequivalent characters $\xi, \zeta$ we will always find $a \in \mathbb{A}$ such that $j_{\xi}(a) \neq j_{\zeta}(a)$.

For each pair $(\xi, \zeta)$ of nonequivalent characters we fix $a$ so that $j_{\xi}(a) \neq$ $j_{\zeta}(a)$. We can now modify the definition of $\psi$ to obtain a subsequence $\left(r_{p}^{(\xi, \zeta)}\right)$ of $\left(r_{0}^{(k)}\right)$ along which we have convergence as in Lemma 11.

Proposition 4. Under all the above assumptions on $T, \varphi$ and $\Theta$, the set of essential values of the multiplicity function of $T_{\varphi} \times\left(T_{\varphi}\right)_{\Theta}$ is equal to

$\{2\} \cup$ the set of lengths of $\widehat{v}$-orbits of nontrivial characters.

Proof. We write $T_{\varphi} \times\left(T_{\varphi}\right)_{\Theta}=\left(T_{\varphi} \times T_{\varphi}\right)_{\widetilde{\Theta}}$. Then

$$
U_{T_{\varphi} \times\left(T_{\varphi}\right)_{\Theta}}=\bigoplus_{\xi \in \widehat{\mathbb{A}}} V_{\widetilde{\Theta}, T_{\varphi} \times T_{\varphi}}^{\xi}, \quad V_{\widetilde{\Theta}, T_{\varphi} \times T_{\varphi}}^{\xi}=U_{T_{\varphi}} \otimes V_{\Theta, T_{\varphi}}^{\xi} .
$$

We claim that

$$
\begin{aligned}
& U_{T_{\varphi}} \otimes U_{T_{\varphi}} \text { has uniform multiplicity } 2 \\
& U_{T_{\varphi}} \otimes V_{\Theta, T_{\varphi}}^{\xi}, \xi \neq 1 \text {, has a simple spectrum; } \\
& \xi \equiv \zeta \text { implies } U_{T_{\varphi}} \otimes V_{\Theta, T_{\varphi}}^{\xi} \text { and } U_{T_{\varphi}} \otimes V_{\Theta, T_{\varphi}}^{\zeta} \text { are isomorphic; } \\
& \xi \not \equiv \zeta \text { implies that the spectral measures of } \\
& U_{T_{\varphi}} \otimes V_{\Theta, T_{\varphi}}^{\xi} \text { and } U_{T_{\varphi}} \otimes V_{\Theta, T_{\varphi}}^{\zeta} \text { are mutually singular. }
\end{aligned}
$$

Indeed, (32) follows from Lemmas 2 and 8. In order to prove (33) we first notice that $V_{\Theta, T_{\varphi}}^{\xi}$ has a simple spectrum. Indeed, this follows from the choice of towers (see (20)) and the fact that the cocycle $\Theta$ is constant on the levels of the corresponding cyclic tower. Moreover,

$$
\left(U_{T_{\varphi}}\right)^{n_{s}^{(0,0,0)}} \rightarrow \frac{1}{2}\left(\mathrm{Id}+U_{T_{\varphi}}\right),
$$

where we consider $U_{T_{\varphi}}$ on the whole space $L^{2}(X, \mathcal{B}, \mu)$ and not only on $L_{0}^{2}(X, \mathcal{B}, \mu)$,

$$
\begin{aligned}
\left(V_{\Theta, T_{\varphi}}^{\xi}\right)^{n_{s}^{(0,0,0)}} & \rightarrow \frac{1}{2}\left(j_{\xi}(0) \cdot \mathrm{Id}+j_{\xi}(0) \cdot V_{\Theta, T_{\varphi}}^{\xi}\right)=\frac{1}{2}\left(\mathrm{Id}+V_{\Theta, T_{\varphi}}^{\xi}\right), \\
\left(U_{T_{\varphi}}\right)^{n_{s}^{(0, a, 0)}} & \rightarrow \frac{1}{2}\left(\mathrm{Id}+U_{T_{\varphi}}\right), \\
\left(V_{\Theta, T_{\varphi}}^{\xi}\right)^{n_{s}^{(0, a, 0)}} & \rightarrow \frac{1}{2}\left(j_{\xi}(0) \cdot \mathrm{Id}+j_{\xi}(a) \cdot V_{\Theta, T_{\varphi}}^{\xi}\right)=\frac{1}{2}\left(\mathrm{Id}+j_{\xi}(a) \cdot V_{\Theta, T_{\varphi}}^{\xi}\right),
\end{aligned}
$$

where $a$ is chosen so that $j_{\xi}(a) \neq 1$ and an application of Lemma 1 completes the proof of (33). The property (34) is obvious and finally to prove (35) we use

$$
\begin{aligned}
\left(U_{T_{\varphi}} \otimes V_{\Theta, T_{\varphi}}^{\xi}\right)^{r_{p}^{(\xi, \zeta)}}= & \left(U_{T_{\varphi}}\right)^{r_{p}^{(\xi, \zeta)}} \otimes\left(V_{\Theta, T_{\varphi}}^{\xi}\right)^{r_{p}^{(\xi, \zeta)}} \rightarrow \operatorname{Id} \otimes j_{\xi}(a) \operatorname{Id}=j_{\xi}(a) \cdot \operatorname{Id}, \\
& \left(U_{T_{\varphi}} \otimes V_{\Theta, T_{\varphi}}^{\zeta}\right)^{r_{p}^{(\xi, \zeta)}} \rightarrow j_{\zeta}(a) \cdot \mathrm{Id}
\end{aligned}
$$


where $j_{\xi}(a) \neq j_{\zeta}(a)$, proving the mutual singularity of the spectral measures.

Proof of Theorem 1. We will now make use of Proposition 4 and of the idea from [19] to pass to so called natural factors and in particular complete the proof of Theorem 1 . For that we just take a closed subgroup $\mathbb{B} \subset \mathbb{A}$ and by considering $U_{\left(T_{\varphi}\right)_{\Theta}}$ on the quotient space $X \times \mathbb{Z}_{m} \times \mathbb{A} / \mathbb{B}$ we count only the cardinalities of the intersections of $w:=\widehat{v}$-orbits with the annihilator subgroup $\mathbb{F}$ of $\mathbb{B}$ in $\mathbb{G}:=\widehat{\mathbb{A}}$ (see [19]) in order to obtain all, other than 2, spectral multiplicities of the factor of $\left(T_{\varphi}\right)_{\Theta}$.

5. Examples of Koopman realizable subsets. We have been unable to show whether or not the following modification of the Algebraic Lemma from [19] holds (it would imply that all subsets containing 2 are Koopman realizable): Assume that $F \subset \mathbb{N}$ is a finite set without 1 . Then there exist a countable Abelian group $\mathbb{G}$, its subgroup $\mathbb{F}$ and an automorphism $w: \mathbb{G} \rightarrow \mathbb{G}$ for which $w^{M}=\operatorname{Id}$ such that $\left\{\sharp\left(\left\{w^{i}(\chi): i \geq 0\right\} \cap \mathbb{F}\right): \chi \in \mathbb{G} \backslash\{0\}\right\}=F$. Therefore we will study only some particular subsets $F$.

Fix $M \geq 2$. Let $\mathbb{G}$ be a countable Abelian group and $w: \mathbb{G} \rightarrow \mathbb{G}$ be its automorphism (by duality, $\mathbb{G}$ and $w$ play the roles of $\widehat{\mathbb{A}}$ and $\widehat{v}$ ) such that for $\chi \in \mathbb{G} \backslash\{0\}$,

$$
\sharp\left\{w^{i}(\chi): i \geq 0\right\}=M
$$

(in particular $w^{M}=\mathrm{Id}$ ); see the example below. By applying Proposition 4 to $\mathbb{A}=\widehat{\mathbb{G}}$ and $v=\widehat{w}$ we obtain the following statement which in particular answers a question from [33].

COROLlary 3. For each $M \geq 2$ there exists a weakly mixing transformation $\bar{T}$ such that the set of spectral multiplicities of $U_{\bar{T}}$ equals $\{2, M\}$.

Let us now give an example of $(\mathbb{G}, w)$ satisfying $(36)$ - it will be a particular case of Lemma 2.1 from [30].

First choose $p$ prime so that $M$ divides $p-1$ (which is possible by the Dirichlet theorem on primes in arithmetic progressions). Our group will be the cyclic group $\mathbb{G}=\mathbb{Z} /(p \mathbb{Z})$ and the automorphism $w$ is defined by $w(g)=$ $a \cdot g$, where $a \in \mathbb{G}$ is chosen in the following way. Notice that the multiplicative group $H=(\mathbb{Z} /(p \mathbb{Z}))^{*}$ is cyclic of order $p-1$; hence it contains a (cyclic) subgroup $I \subset H$ of order $M$. Take $a \in I$ to be its generator. The orbits of $w$ are of the form $I \cdot g$, i.e. the (multiplicative) cosets of $I$ in $H$, so (because all elements in $\mathbb{G} \backslash\{0\}$ are invertible, or just using the fact that $\mathbb{Z} /(p \mathbb{Z})$ is a field) all orbits of nonzero elements have cardinality equal to that of $I$.

Now take $M \geq 2$ and arbitrary $k \geq 2$. We will show how to Koopman realize the set

$$
\{2, k, k M\}=\{2\} \cup M \cdot\{1, k\} .
$$


To this end take, as before, a countable Abelian group $\mathbb{G}$ and an automorphism $w: \mathbb{G} \rightarrow \mathbb{G}$ such that the $w$-orbits of all $\xi \in \mathbb{G} \backslash\{0\}$ have length $M$. Consider $\overline{\mathbb{G}}=\mathbb{G}^{\times S}$ where $S \geq 1$ is a large number of the form $t M+1$. Now take $1=m_{1}<\cdots<m_{k}$ so that for each $i>0$,

$$
\sharp\left(\left(\left\{m_{r}: r=1, \ldots, k\right\}+i\right) \bmod S \cap\left\{m_{r}: r=1, \ldots, k\right\}\right) \leq 1 .
$$

Put

$$
\mathbb{F}=\left\{\bar{\chi}=\left(\chi_{i}\right)_{i=1}^{S} \in \overline{\mathbb{G}}: \chi_{i}=0 \text { for } i \neq m_{r}, r=1, \ldots, k\right\}
$$

Define

$$
u\left(\chi_{1}, \ldots, \chi_{S}\right)=\left(w\left(\chi_{S}\right), w\left(\chi_{1}\right), \ldots, w\left(\chi_{S-1}\right) .\right.
$$

It is now easy to see that the $u$-orbit of $\bar{\chi}$ for which $\chi_{i} \neq 0$ for only one $i$, meets $\mathbb{F}$ exactly $M \cdot k$ times, while if $\chi_{i} \neq 0 \neq \chi_{j}$ for some $i<j$, then the $u$-orbit of $\chi$ either meets $\mathbb{F}$ exactly $M$ times, or does not meet $F$ at all.

Given $k \geq 2$ and a finite set $F \subset \mathbb{N}$ without $1, F=\left\{k_{1}, \ldots, k_{f}\right\}$, the above construction can be extended similarly to [19] by considering $\widetilde{\mathbb{G}}=\bar{G}_{1} \times$ $\cdots \times \bar{G}_{f}$ where $\bar{G}_{j}=\mathbb{G}^{\times S}, j=1, \ldots, f$; we choose however $m_{1}^{(j)}<\cdots<m_{k_{j}}^{(j)}$ satisfying (37) so that for each $i \geq 0$ and $j \neq j^{\prime}$,

$$
\sharp\left(\left(\left\{m_{r}^{(j)}: r=1, \ldots, k\right\}+i\right) \bmod S \cap\left\{m_{r}^{\left(j^{\prime}\right)}: r=1, \ldots, k\right\}\right) \leq 1 ;
$$

$\widetilde{\mathbb{F}}$ is defined to be the product of the corresponding $\mathbb{F}_{j}$ s. The set $M(\widetilde{\mathbb{G}}, \widetilde{u}, \widetilde{\mathbb{F}})$ of orbits "induced" on $\widetilde{\mathbb{F}}$ will then be equal to $M \cdot\left\{1, k_{1}, \ldots, k_{f}\right\}$. Therefore we obtain the following corollary which is to be compared with Danilenko's spectral multiplicity result from [8].

COROLlary 4. For each finite subset $F$ of $\mathbb{N}$ without 1 and $M \geq 1$ there exists a weakly mixing transformation $R$ such that the set of essential values of the multiplicity function of $U_{R}$ equals $\{2\} \cup M \cdot(\{1\} \cup F)$.

\section{Some speculations}

6.1. On spectral multiplicity. In order to obtain other Koopman realizable sets we can use Proposition 1 to compute the spectral multiplicities of $T_{\varphi}^{\times r} \times\left(T_{\varphi}\right)_{\Theta}(r \geq 2)$. In this case we need to use the fact that a "typical" automorphism admits a linked approximation $(n, n+1)$ with proportions of the towers $0<\kappa<1$ for infinitely many $\kappa$ s and we must repeat all the proofs with computations of limit operators for the double extension. In this way we could obtain Koopman realization of the sets of the form

$$
\{k, k(k-1), \ldots, k !\} \cup\{k-1,(k-1)(k-2), \ldots,(k-1) !\} \cdot M(\mathbb{G}, w, \mathbb{F}) .
$$

For example, taking $k=3$ we obtain $\{3,6\} \cup 2 \cdot M(\mathbb{G}, w, \mathbb{F})$.

Finally, analysis of spectral multiplicities for "natural" factors of Cartesian products $T_{\varphi}^{\times r}$ can also be applied (see $[16,5]$ ). 
Passing to a more general discussion notice that a generic measure preserving transformation has a simple spectrum and virtually all natural constructions lead to spectrum of infinite multiplicity. Appearance of nonsimple finite multiplicity spectrum in all known constructions is due to a certain symmetry. Three basic types of symmetry used in this context are:

1. symmetry of double skew products with a group structure in the second extension, first noticed by Oseledets [27], originally systematically explored by Robinson [29, 30] and further developed in [12],

2. the obvious symmetry of the Cartesian powers, first used in the unpublished version of [16] which has circulated since mid-eighties, and brought to the final form in $[1,32]$, and

3. symmetry involving a certain non-Abelian finite extension of a cyclic group discovered by Ageev [4].

There are various simple derivatives such as taking factors of Cartesian powers. The point of the present paper is to combine constructions of type 1 and 2 in a rather sophisticated way. One can systematically explore what various combinations of these three basic methods give but certain fairly simple cases of spectral multiplicity are still not covered and realization of those cases should wait for the discovery of new types of symmetry. Among the simplest unresolved cases are $\{3,4\},\{3,5\}$ and $\{3,7\}$.

6.2. On maximal spectral type. Once one passes from the pure multiplicity question where restrictions (and hence "real theorems") are unlikely, to other problems of spectral realization the situation begins to look quite bleak. There is a huge gap between basic restrictions on the maximal spectral type and known cases of realization; among the latter, Gaussian systems give realization of the type of any measure which is the union of an arbitrary symmetric measure on $\mathbb{T}$ and its convolutions of all orders. In certain cases maximal spectral type can be calculated as a Riesz product [20], [26, Chapter 16].

It is hard to think of a naturally formulated restriction on a continuous maximal spectral type beyond density and symmetry, although possibly an indirect argument based on looking at multiplicative unitary operators among all unitary operators in $L_{2}$ on a standard Borel space, may produce nonexplicit existence of a nonrealizable type. What is known is that many specific properties of the maximal spectral type are realizable, such as mutual singularity of the maximal spectral type and all its convolutions (see e.g. [16]), or arbitrary "thinness" of the maximal spectral type (arbitrary sharp concentration around certain roots of unity), but on the other hand, there are striking open questions:

Problem 1. Can the maximal spectral type be absolutely continuous but not Lebesgue? 
There is a chance for a negative answer here which would produce a real "theorem". If one believes in a positive answer, here is a simpler question:

Problem 2. Can a measure of maximal spectral type $\sigma$ for $U_{T}$ be absolutely continuous with respect to its convolution $\sigma * \sigma$ but not equivalent to $i t$ ?

Notice that there are three known possibilities:

1. $\sigma$ is equivalent to $\sigma * \sigma$, as for Lebesgue spectrum or for Gaussian systems;

2. $\sigma$ and $\sigma * \sigma$ are mutually singular, as for a generic measure preserving transformation $T$;

3. $\sigma$ and $\sigma * \sigma$ have a common part but neither is absolutely continuous with respect to the other, as for $T \times T$ for a generic $T$.

6.3. On other problems. When one tries to combine the maximal spectral type and multiplicity, even basic problems remain open. Here is another chance for a theorem. Recall that an equivalent description of the spectral invariants for a unitary operator includes instead of the multiplicity function $M$ the decreasing sequence of spectral types $\sigma_{n}$ corresponding to the restrictions of the maximal spectral type to the sets $M^{-1}([n, \infty) \cup\{\infty\})$, $n \in \mathbb{N}^{*} \cup\{\infty\}$.

PROBLem 3. Is it true that all spectral types of a measure preserving transformation with continuous spectrum are dense?

The reader is unquestionably familiar with the celebrated problem of simple Lebesgue spectrum. Here is something one can try if one believes that examples are more likely than theorems.

Problem 4. Does there exist an ergodic measure preserving transformation whose maximal spectral type is absolutely continuous but the spectrum is not Lebesgue with countable multiplicity?

The difference from Problem 1 is that it is conceivable that the maximal spectral type is Lebesgue while not all others are; this is motivated by known examples with Lebesgue component of finite multiplicity in the spectrum (see e.g. $[26])$.

Let us finish by saying that the above remarks going beyond the spectral multiplicity problem by no means pretend to be an overview of the subject or even to present a coherent thinking about it. We just wanted to illustrate the rather unsatisfactory state of the subject and offer a sample of characteristic open questions.

7. Appendix: Lifting weak convergences from $T$ to $T_{\varphi, \mathcal{S}}$. This part is not directly related to the main subject of the paper. We will show 
here that the method of lifting weak convergences to extensions given by "ordinary" cocycles which we have seen in previous sections may also be used in the case of some Rokhlin cocycles considered recently in [21] and [22]. This will allow us to answer a question implicitly contained in [23].

Assume that $T$ is an ergodic transformation that admits a linked approximation of type $(n, n+1)$ as in the previous section; this means that there is a (nested) sequence of generating partitions (C). In particular, (19) holds.

Let $\mathbb{A}$ be an l.c.s.c. Abelian group. We will now define certain cocycles with values in $\mathbb{A}$ for $T$ admitting linked $(n, n+1)$ approximation. The construction will follow the previously described general scheme: We assume that $\varphi$ is defined on all levels $T^{i} C_{t}$ (taking constant values on such levels) except for some levels including the level $T^{n_{t}-1} C_{t}$. Then we put arbitrary constant values of $\varphi$ on $T^{i} C_{t}\left(0 \leq i \leq n_{t}-2\right)$ where $\varphi$ has not been defined yet. Next, such a tower is divided into subcolumns which also refine the subtowers based on $C_{t}^{1}$ and $C_{t}^{2}$ respectively. We put constant values of $\varphi$ on all top levels of the subcolumns contained in the tower with bottom $C_{t}^{1}$, while for subcolumns contained in the other subtower we put constant values of $\varphi$ on the top level and its image via $T$; we keep $\varphi$ undefined only on the image via $T$ of the top of the last subcolumn.

Assume now that $\mathbb{A}$ has a (faithful) measurable representation $a \mapsto S_{a}$ in $\operatorname{Aut}(Y, \mathcal{C}, \nu)$. Given a cocycle $\varphi: X \rightarrow \mathbb{A}$ let $T_{\varphi, \mathcal{S}}$ stand for the automorphism of $(X \times Y, \mathcal{B} \otimes \mathcal{C}, \mu \otimes \nu)$ given by

$$
T_{\varphi, \mathcal{S}}(x, y)=\left(T x, S_{\varphi(x)}(y)\right) .
$$

Notice that $\mathbb{A}$ acts on $(X \times Y, \mu \otimes \nu)$ by $\operatorname{Id}_{X} \times S_{a}$ and moreover that $\operatorname{Id}_{X} \times S_{a} \in$ $C\left(T_{\varphi, \mathcal{S}}\right)$.

Let us now fix $b, c \in \mathbb{A}$. We will assume that for some subsequence of $\left(n_{t}\right)$ (for simplicity still denoted by $\left(n_{t}\right)$ ) we have

$$
\begin{gathered}
\varphi^{\left(n_{t}\right)}(x)=b \quad \text { for } x \in \bigcup_{i=0}^{n_{t}-1} T^{i} C_{t}^{1} \\
\varphi^{\left(n_{t}+1\right)}(x)=c \quad \text { for } x \in \bigcup_{i=0}^{n_{t_{k}}} T^{i} C_{t}^{2} \text { except in the last subcolumn. }
\end{gathered}
$$

Proposition 5. Under the above assumptions,

$$
\left(U_{T_{\varphi, \mathcal{S}}}\right)^{n_{t}+1} \rightarrow \frac{1}{2}\left(U_{\mathrm{Id}_{X} \times S_{c}}+U_{T_{\varphi, \mathcal{S}} \circ\left(\operatorname{Id}_{X} \times S_{b}\right)}\right)
$$

weakly as Markov operators on $L^{2}(X \times Y, \mu \otimes \nu)$. In particular, if $b=c=0$ then the weak convergence (19) holds for $T_{\varphi, \mathcal{S}}$. 
Proof. Take $f \in L^{\infty}(X, \mathcal{B}, \mu), g \in L^{\infty}(Y, \mathcal{C}, \nu)$ and put $H(x, y)=f(x) g(y)$. Then

$$
\begin{array}{r}
\int_{X \times Y} H \circ T_{\varphi, \mathcal{S}}^{n_{t}+1} \cdot \bar{H} d \mu d \nu=\int_{X \times Y} H\left(T^{n_{t}+1} x, S_{\varphi^{\left(n_{t}+1\right)}(x)}(y)\right) \overline{H(x, y)} d \mu(x) d \nu(y) \\
=\int_{X} f\left(T^{n_{t}+1} x\right) \overline{f(x)}\left(\int_{Y} g\left(S_{\varphi^{\left(n_{t}+1\right)}}(y)\right) \overline{g(y)} d \nu(y)\right) d \mu(x) .
\end{array}
$$

Replace $f$ by $f_{t}$ so that $\left\|f_{t}\right\|_{\infty} \leq\|f\|_{\infty}$ and $\left\|f-f_{t}\right\|_{L^{2}}<\varepsilon /\|g\|_{\infty}$, where $f(x)=f_{i}$ for all $x \in T^{i} C_{t}, i=0,1, \ldots, n_{t}-1$. Put $H_{t}=f_{t} \otimes g$ and notice that $\left\|H_{t}-H\right\|_{L^{2}}<\varepsilon$ and $\left\|H_{t}\right\|_{\infty} \leq\|H\|_{\infty}$. Therefore

$$
\begin{aligned}
& \left\|\int_{X \times Y} H \circ T_{\varphi, \mathcal{S}}^{n_{t}+1} \bar{H} d \mu d \nu-\int_{X \times Y} H_{t} \circ T_{\varphi, \mathcal{S}}^{n_{t}+1} \overline{H_{t}} d \mu d \nu\right\| \\
& \quad \leq\left\|H-H_{t}\right\|_{L^{2}}\|\bar{H}\|_{\infty}+\left\|H_{t}\right\|_{\infty}\left\|\bar{H}-\bar{H}_{t}\right\|_{L^{2}}<2 \varepsilon\|f\|_{\infty} .
\end{aligned}
$$

This argument uses only the fact that our functions are bounded and that the transformations we use are measure preserving. In what follows we will apply this approximation argument without detailed justification.

Define $\widetilde{C^{1}}=\bigcup_{i=0}^{n_{t}-1} T^{i} C_{t}^{1}$ and $\widetilde{C^{2}}=\bigcup_{i=0}^{n_{t}} T^{i} C_{t}^{2}$. Using the Spectral Theorem, Fubini's Theorem and properties of $\varphi$ and $f_{t}$ (they are constant on the levels $T^{i} C_{t}$ ) we have

$$
\begin{aligned}
\int_{X \times Y} H_{t} \circ T_{\varphi, \mathcal{S}}^{n_{t}+1} & \cdot \bar{H}_{t} d \mu d \nu \\
= & \int_{X} f_{t}\left(T^{n_{t}+1} x\right) \overline{f_{t}(x)}\left(\int_{Y} g\left(S_{\varphi^{\left(n_{t}+1\right)}}(y)\right) \overline{g(y)} d \nu(y)\right) d \mu(x) \\
= & \int_{X} f_{t}\left(T^{n_{t}+1} x\right) \overline{f_{t}(x)}\left(\int_{\widehat{G}} \chi\left(\varphi^{\left(n_{t}+1\right)}(x)\right) d \sigma_{g, \mathcal{S}}(\chi)\right) d \mu(x) \\
= & \int_{\widehat{G}}\left(\int_{X} \chi\left(\varphi^{\left(n_{t}+1\right)}(x)\right) f_{t}\left(T^{n_{t}+1} x\right) \overline{f_{t}(x)} d \mu(x)\right) d \sigma_{g, \mathcal{S}}(\chi) \\
= & \int_{\widehat{G}}\left(\int_{\widetilde{C}^{1}} \chi\left(\varphi^{\left(n_{t}\right)}(x)\right) \chi\left(\varphi\left(T^{n_{t}} x\right)\right) f_{t} \circ T\left(T^{n_{t}} x\right) \overline{f_{t}(x)} d \mu(x)\right. \\
& \left.+\int_{\widetilde{C}^{2}} \chi\left(\varphi^{\left(n_{t}+1\right)}(x)\right) f_{t}\left(T^{n_{t}+1} x\right) \overline{f_{t}(x)} d \mu(x)\right) d \sigma_{g, \mathcal{S}}(\chi)+\mathrm{o}(1) \\
= & \int_{\widehat{G}}\left(\sum_{i=0}^{n_{t}-1} \int_{T^{i} C_{t}^{1}} \chi(b) \chi(\varphi(x)) f_{t, i+1} \bar{f}_{t, i} d \mu(x)\right. \\
& \left.+\sum_{i=0}^{n_{t}} \int_{T^{i} C_{t}^{2}} \chi(c) f_{t, i} \bar{f}_{t, i} d \mu(x)\right) d \sigma_{g, \mathcal{S}}(\chi)+\mathrm{o}(1)+\mathrm{o}(1)
\end{aligned}
$$




$$
\begin{aligned}
= & \int_{\widehat{G}}\left(\int_{\widetilde{C}^{1}} \chi(\varphi(x)+b) f_{t}(T x) \bar{f}_{t}(x) d \mu(x)\right. \\
& \left.+\int_{\widetilde{C}^{2}} \chi(c) f_{t}(x) \bar{f}_{t}(x) d \mu(x)\right) d \sigma_{g, \mathcal{S}}(\chi)+\mathrm{o}(1)+\mathrm{o}(1) \\
= & \int_{\widehat{G}}\left(\frac{1}{2} \int_{X} \chi(\varphi(x)+b) f_{t}(T x) \bar{f}_{t}(x) d \mu(x)\right. \\
& \left.+\frac{1}{2} \int_{X} \chi(c) f_{t}(x) \bar{f}_{t}(x) d \mu(x)\right) d \sigma_{g, \mathcal{S}}(\chi)+\mathrm{o}(1)+\mathrm{o}(1)+\mathrm{o}(1) \\
= & \frac{1}{2}\left(\int_{X \times Y} H_{t} \circ T_{\varphi, \mathcal{S}} \circ\left(\operatorname{Id} \times S_{b}\right) \overline{H_{t}} d \mu d \nu\right. \\
& \left.+\int_{X \times Y} H_{t} \circ\left(\operatorname{Id} \times S_{c}\right) \overline{H_{t}} d \mu d \nu\right)+\mathrm{o}(1)+\mathrm{o}(1)+\mathrm{o}(1)+\mathrm{o}(1) .
\end{aligned}
$$

Therefore

$$
\begin{aligned}
\int_{X \times Y} H \circ T_{\varphi, \mathcal{S}}^{n_{t}+1} \cdot \bar{H} d \mu d \nu & =\frac{1}{2}\left(\int_{X \times Y} H \circ T_{\varphi, \mathcal{S}} \circ\left(\operatorname{Id} \times S_{b}\right) \bar{H} d \mu d \nu\right. \\
& \left.+\int_{X \times Y} H \circ\left(\operatorname{Id} \times S_{c}\right) \bar{H} d \mu d \nu\right)+\mathrm{o}(1)+\mathrm{o}(1)+\mathrm{o}(1)+\mathrm{o}(1)+\mathrm{o}(1),
\end{aligned}
$$

and the result follows.

In the whole paper (except for this section) we have dealt with compact group extensions of transformations. Such extensions are special cases of so called distal extensions and the complementary notion is that of relative weak mixing [10]. In fact, in the case of transformations of the form $T_{\varphi, \mathcal{S}}$ the extension $T_{\varphi, \mathcal{S}} \rightarrow T$ is in general relatively weakly mixing.

To deal with the relatively weakly mixing case, following [21], our first task is to show that we can get $\varphi: X \rightarrow \mathbb{A}$ ergodic. In order to proceed we will assume additionally that the linked approximation is mixed with a cyclic approximation (i.e. (B) and (C) hold), and altogether we obtain a nested sequence of towers. "Practically", having a sequence of towers, at an even stage of the construction we divide into many subcolumns and add no spacers for the first half of subcolumns, and add exactly one spacer for the remaining subcolumns, while for odd stages we do not add spacers (this means we add "few" spacers). Now to lift weak convergence we will use $(n, n+1)$-towers, while rigid towers will be used to get ergodicity of $\varphi$. For example, to guarantee ergodicity we will have our cocycle constant on the levels of the rigid tower except for the last level, and then for this last level we make the sum close to a fixed element of the group while few spacers 
which we add will not affect this property. Now a criterion for ergodicity from [25] guarantees ergodicity of $\varphi$. Notice that the construction of $\varphi$ on rigid towers is compatible with the construction of $\varphi$ on linked towers.

If now $\varphi: X \rightarrow \mathbb{A}$ is ergodic, and $\mathcal{S}$ is mildly mixing, then $T_{\varphi, \mathcal{S}}$ is relatively weakly mixing over $T[21]$.

In [23] a spectral notion of 2-fold singular convolutions (SC) has been introduced. By definition, an ergodic automorphism $T$ has this property if its (reduced) maximal spectral type is singular with respect to the convolution of any two continuous measures. The 2-fold SC property implies some strong joining property of $T$ with Cartesian product transformations similar to properties of so called distally simple automorphisms [14]. A natural question arises whether the 2 -fold SC property implies distal simplicity. However, as already noticed in [23], the condition (19) implies 2-fold SC property. Moreover, when we carry out the construction of $\varphi: X \rightarrow \mathbb{A}$ as above and we apply Proposition 5 we find that (19) holds for $T_{\varphi, \mathcal{S}}$, so $T_{\varphi, \mathcal{S}}$ also has the 2 -fold SC property. However, it is not distally simple, as a distally simple automorphism is a distal extension of any of its nontrivial factors [14], while $T_{\varphi, \mathcal{S}}$ is a relatively weakly mixing extension of $T$.

COROLlary 5. For a typical automorphism $T$ there exists a cocycle $\varphi: X \rightarrow \mathbb{A}$ such that for each mildly mixing $\mathbb{A}$-representation $a \mapsto S_{a}$ in Aut $(Y, \mathcal{C}, \nu)$ the automorphism $T_{\varphi, \mathcal{S}}$ has the 2 -fold $S C$ property but is not distally simple. In fact, the extension $T_{\varphi, \mathcal{S}} \rightarrow T$ is relatively weakly mixing.

Acknowledgements. We would like to thank the referee for making useful suggestions that led to an improvement of presentation in several places.

Research of A. Katok was partially supported by NSF Grants DMS 0505539 and 0803880 , and by Marie Curie "Transfer of Knowledge" EU program, project MTKD-CT-2005-030042 (TODEQ).

Research of M. Lemańczyk was partially supported by Polish MNiSzW grant N N201 384834 and by Marie Curie "Transfer of Knowledge" EU program, project MTKD-CT-2005-030042 (TODEQ).

\section{References}

[1] O. N. Ageev, On ergodic transformations with homogenous spectrum, J. Dynam. Control Systems 5 (1999), 149-152.

[2] - On the spectrum of Cartesian powers of classical automorphisms, Mat. Zametki 68 (2000), 643-647 (in Russian); English transl.: Math. Notes 68 (2000), 547-551.

[3] - On the multiplicity function of generic group extensions with continuous spectrum, Ergodic Theory Dynam. Systems 21 (2001), 321-338.

[4] - , The homogeneous spectrum problem in ergodic theory, Invent. Math. 160 (2005), 417-446. 
[5] O. N. Ageev, Mixing with staircase multiplicity function, Ergodic Theory Dynam. Systems 28 (2008), 1687-1700.

[6] D. V. Anosov, On spectral multiplicities in ergodic theory, in: Current Problems in Mathematics 3, Rossiǔskaya Akademiya Nauk, Mat. Inst. im. V. A. Steklova, Moscow, 2003, 86 pp. (in Russian); http://www.mi.ras.ru/spm/pdf/003.pdf.

[7] I. P. Cornfeld, S. V. Fomin, and Y. G. Sinai, Ergodic Theory, Springer, New York, 1982.

[8] A. Danilenko, Explicit solution of Rokhlin's problem on homogenous spectrum and applications, Ergodic Theory Dynam. Systems 26 (2006), 1467-1490.

[9] Y. Derriennic, K. Frączek, M. Lemańczyk, and F. Parreau, Ergodic automorphisms whose weak closure of off-diagonal measures consists of ergodic self-joinings, Colloq. Math. 110 (2008), 81-115.

[10] H. Furstenberg, Recurrence in Ergodic Theory and Combinatorial Number Theory, Princeton Univ. Press, Princeton, NJ, 1981.

[11] G. R. Goodson, A survey of recent results in the spectral theory of ergodic dynamical systems, J. Dynam. Control Systems 5 (1999), 173-226.

[12] G. R. Goodson, J. Kwiatkowski, M. Lemańczyk, and P. Liardet, On the multiplicity function of ergodic group extensions of rotations, Studia Math. 102 (1992), 157-174.

[13] M. Guenais, Une majoration de la multiplicité spectrale d'opérateurs associés à des cocycles réguliers, Israel J. Math. 105 (1998), 263-283.

[14] A. del Junco and M. Lemańczyk, Distally simple systems, preprint, 2005.

[15] A. B. Katok, Monotone equivalence in ergodic theory, Izv. Akad. Nauk SSSR Ser. Mat. 41 (1977), 104-157 (in Russian).

[16] -, Combinatorial Constructions in Ergodic Theory and Dynamics, Univ. Lecture Ser. 30, Amer. Math. Soc., 2003.

[17] A. B. Katok and A. M. Stepin, Approximations in ergodic theory, Uspekhi Mat. Nauk 22 (1967), no. 5, 81-106 (in Russian).

[18] A. Katok and J.-P. Thouvenot, Spectral properties and combinatorial constructions in ergodic theory, in: Handbook of Dynamical Systems, Vol. 1B, Elsevier, Amsterdam, 2006, 649-743.

[19] J. Kwiatkowski (jr.) and M. Lemańczyk, On the multiplicity function of ergodic group extensions. II, Studia Math. 116 (1995), 207-215.

[20] F. Ledrappier, Des produits de Riesz comme mesures spectrales, Ann. Inst. H. Poincaré 6 (1970), 335-344.

[21] M. Lemańczyk and E. Lesigne, Ergodicity of Rokhlin cocycles, J. Anal. Math. 85 (2001), 43-86.

[22] M. Lemańczyk and F. Parreau, Rokhlin extensions and lifting disjointness, Ergodic Theory Dynam. Systems 23 (2003), 1525-1550.

[23] M. Lemańczyk, F. Parreau, and E. Roy, Systems with simple convolutions, distal simplicity and disjointness with infinitely divisible systems, preprint, 2007.

[24] M. Lemańczyk, F. Parreau, and J.-P. Thouvenot, Gaussian automorphisms whose ergodic self-joinings are Gaussian, Fund. Math. 164 (2000), 253-293.

[25] M. Lemańczyk, F. Parreau, and D. Volný, Ergodic properties of real cocycles and pseudo-homogeneous Banach spaces, Trans. Amer. Math. Soc. 348 (1996), 49194938.

[26] M. G. Nadkarni, Spectral Theory of Dynamical Systems, Birkhäuser, Berlin, 1998.

[27] V. I. Oseledets, On the spectrum of ergodic automorphisms, Soviet Math. Dokl. 7 (1966), 776-779.

[28] W. Parry, Topics in Ergodic Theory, Cambridge Tracts in Math. 75, Cambridge Univ. Press, Cambridge, 1981. 
[29] E. A. Robinson, Ergodic measure preserving transformations with arbitrary finite multiplicities, Invent. Math. 72 (1983), 299-314.

[30] -, Transformations with highly nonhomogeneous spectrum of finite multiplicity, Israel J. Math. 56 (1986), 75-88.

[31] E. Roy, Mesures de Poisson, infinie divisibilité et propriétés ergodiques, Thèse de doctorat, Univ. Paris 6, 2005.

[32] V. V. Ryzhikov, Transformations having homogeneous spectra, J. Dynam. Control Systems 5 (1999), 145-148.

[33] - Weak limits of powers, the simple spectrum of symmetric products, and mixing constructions of rank 1, Mat. Sb. 198 (2007), 137-159 (in Russian); English transl.: Sb. Math. 198 (2007), 733-754.
A. Katok
Department of Mathematics
The Pennsylvania State University
University Park, PA 16802, U.S.A.
E-mail: katok_a@math.psu.edu

M. Lemańczyk

Faculty of Mathematics and Computer Science

Nicolaus Copernicus University

Chopina 12/18

87-100 Toruń, Poland

E-mail: mlem@mat.uni.torun.pl

Received 25 November 2008;

in revised form 12 March 2009 